\title{
Hazes and clouds in a singular triple vortex in Saturn's atmosphere from HST/WFC3 multispectral imaging
}

\author{
J.F. Sanz-Requena ${ }^{\mathrm{a}, \mathrm{b}, *}$, S. Pérez-Hoyos ${ }^{\mathrm{c}}$, A. Sánchez-Lavega ${ }^{\mathrm{c}}$, T. del Rio-Gaztelurrutia ${ }^{\mathrm{c}}$, \\ Patrick G.J. Irwin ${ }^{\mathrm{d}}$ \\ ${ }^{a}$ Departamento de Ciencias experimentales, Universidad Europea Miguel de Cervantes, Valladolid, Spain \\ ${ }^{\mathrm{b}}$ Departamento de Física Teórica, Atómica y Optica, Universidad de Valladolid, Spain \\ ${ }^{\mathrm{c}}$ Departamento de Física Aplicada I, Escuela Técnica Superior de Ingeniería, Universidad del País Vasco, Bilbao, Spain \\ ${ }^{\mathrm{d}}$ Atmospheric, Oceanic and Planetary Physics, University of Oxford, Clarendon Laboratory, Parks Road, Oxford OX1 3PU, UK
}

\section{A R T I C L E I N F O}

\section{Keywords:}

Saturn

Saturn atmospheres

Radiative transfer

Hubble space telescope observations

\begin{abstract}
A B S T R A C T
In this paper we present a study of the vertical haze and cloud structure over a triple vortex in Saturn's atmosphere in the planetographic latitude range $55^{\circ} \mathrm{N}-69^{\circ} \mathrm{N}$ (del Río-Gaztelurrutia et al., 2018) using HST/WFC3 multispectral imaging. The observations were taken during 29-30 June and 1 July 2015 at ten different filters covering spectral range from the $225 \mathrm{~nm}$ to $937 \mathrm{~nm}$, including the deep methane band at $889 \mathrm{~nm}$. Absolute reflectivity measurements of this region at all wavelengths and under a number of illumination and observation geometries are fitted with the values produced by a radiative transfer model. Most of the reflectivity variations in this wavelength range can be attributed to changes in the tropospheric haze. The anticyclones are optically thicker $(\tau \sim 25$ vs $\sim 10$ ), more vertically extended ( $\sim 3$ gas scale heights vs $\sim 2)$ and their bases are located deeper in the atmosphere (550 mbar vs 500 mbar) than the cyclone.
\end{abstract}

\section{Introduction}

The zonal wind profile of Saturn's upper clouds is approximately symmetrical, with a strong prograde equatorial jet and four other eastward jets in the northern and southern hemispheres (SánchezLavega et al., 2000; García-Melendo et al., 2011). The jet at $65^{\circ} \mathrm{N}$ planetographic latitude (PG) (in this paper, all latitudes are given in planetographic units, except stated otherwise) has a singular structure, with a double peak (del Río-Gaztelurrutia et al., 2018; Del Genio et al., 2009) that marks two different dynamical regions that are very close in latitude. Both have a similarly high eastward velocity and the ambient vorticity facilitates the coupling of opposite voriticity ovals located to the north and south of the velocity local minimum, as shown in Fig. 1 (del Río-Gaztelurrutia et al., 2018). This double jet seems to be permanent having been observed since Voyager times (Sánchez-Lavega et al., 2000; García-Melendo et al., 2011).

In fact, at the latitude of this double peak, a system of three vortices, a cyclone and two anticyclones can be tracked in Cassini ISS images since the beginning of 2012 (del Río-Gaztelurrutia et al., 2018), confirming that vortices in Saturn can be long-lived (Trammell et al., 2016; del Río-Gaztelurrutia et al., 2010). We shall refer to the triple vortex system as Anticyclone-Cyclone-Anticyclone abbreviated as the ACA system.

In Saturn, the detection of vortices using ground-based telescopes used to be complicated (del Río-Gaztelurrutia et al., 2018), and most of our knowledge of these systems comes from space-based observations. Vortices were first detected during the Voyager flybys in 1980-81 (Smith, 1981, 1982; Ingersoll et al., 1984; García-Melendo et al., 2007), and then by the Hubble Space Telescope (HST) (Sánchez-Lavega et al., 2004) and the Cassini spacecraft (Vasavada et al., 2006; Trammell et al., 2016; Ingersoll et al., 2018; Sayanagi et al., 2019; SánchezLavega et al., 2019a). In more recent times, the improvement of observation techniques has allowed the observation of vortices from Earth even with small sized telescopes and in May 2015, amateur observers detected a disturbance that started at the location of the triple vortex system, which had been previously observed in their images as a dark spot. The perturbation evolved fast, extending rapidly in longitude. The orbits of the Cassini spacecraft at the time were not favorable for the observation of the region, and so we were granted Director Discretionary Time at the Hubble Space Telescope (HST) to observe the region before the perturbation faded away (del Río-Gaztelurrutia et al., 2018). More recently, in 2018, a convective outbreak occurred in the cyclonic side of the poleward jet disturbing the latitude band from $\sim 65^{\circ} \mathrm{N}$ to $76^{\circ} \mathrm{N}$ (Sánchez-Lavega et al., 2019b).

\footnotetext{
* Corresponding author at: Departamento de Ciencias experimentales, Universidad Europea Miguel de Cervantes, Valladolid, Spain.

E-mail address: jfsanz@uemc.es (J.F. Sanz-Requena).
} 


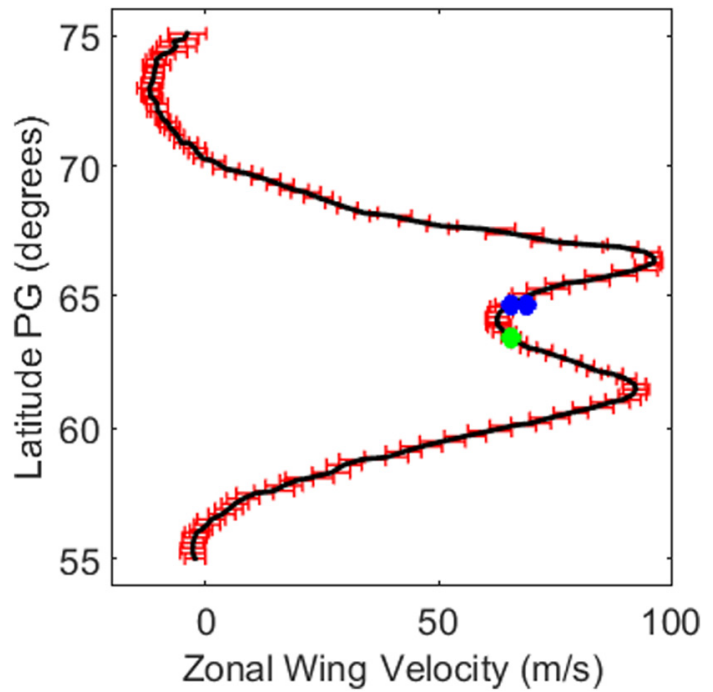

Fig. 1. Zonal wind profile for the region of interest (del Río-Gaztelurrutia et al., 2018). The blue points indicate the location of the anticyclones and the green point indicates the location of the cyclone. (For interpretation of the references to color in this figure legend, the reader is referred to the web version of this article.)

The study of the existence of long-lived vortices and their evolution is an excellent way to increase our understanding of the atmospheric conditions below the observable upper clouds (García-Melendo et al., 2007; del Río-Gaztelurrutia et al., 2010). An essential factor in understanding the atmospheric dynamics of vortices is the knowledge of the vertical distribution of the haze and clouds used as tracers, and to achieve this knowledge we need to determine also the physical and optical properties of the haze particles and clouds in Saturn's stratosphere and upper troposphere (Sanz-Requena et al., 2018). Our current understanding of Saturn's clouds and hazes is constrained by several decades of remote sensing data (e.g., Pérez-Hoyos et al., 2005; Karkoschka and Tomasko, 2005; West et al., 2009) and a usual model is to consider a three-layered aerosol structure formed by a thin stratospheric haze and a denser tropospheric haze, both above a thick cloud layer (Roman et al., 2013).

The goal of this paper is to evaluate the structure of the clouds and hazes and the distribution of aerosols and particles and their properties in the upper troposphere and lower stratosphere in the region of the triple vortex and its environs area, using HST/WFC3 multispectral imaging.

The paper is organized as follows: Section 2 is devoted to a short description of the observations used in this work. Section 3 covers the radiative transfer model, including a description of the vertical cloud structure model and its a priori assumptions. Results are presented and discussed in Section 4, including an analysis of the sensitivity to the model parameters. Results are discussed in Section 5 in terms of the local dynamics and a summary of the main conclusions of this work is presented in Section 6.

\section{Observations}

\subsection{Description of the observations}

In this study, we have used 42 images taken with the Wide Field Camera 3 (WFC3) onboard HST. The images were taken with a variety of filters in three different orbits, on June 29-30 and July 1, 2015. We show in Fig. 2 a representative set of these images.

Table 1 summarizes the observations used in this work and some of the geometric parameters that characterize them. All images have been photometrically calibrated (Dressel, 2019), and navigated and cylindrically projected with the LAIA software, developed by J.A. Cano (Grup d'Estudis Astronomics, GEA) (Sanz-Requena et al., 2012 and Pérez-Hoyos et al., 2005). We assumed a $10 \%$ error in absolute calibration (Dressel, 2019), without taking into account whether these are random or systematic or even known variations from one filter to the other. It must be noted that systematic errors, particularly those regarding absolute calibration, are usually substantially higher than random errors. The former ones can reach up to $10-20 \%$, while the latter ones can be assumed to be always around $1 \%$. This will be of interest later on, when the fitting algorithm is described in Section 3.3.

The filters used in this work are F225W, F336W, F410M, F502N,
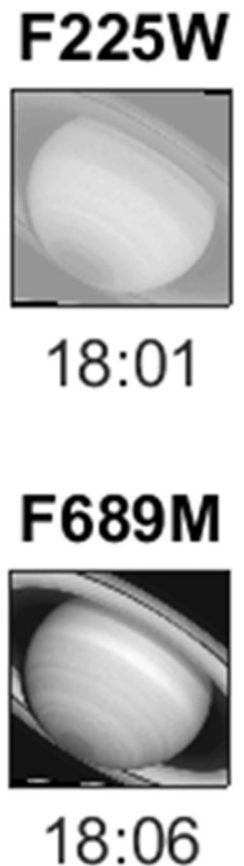
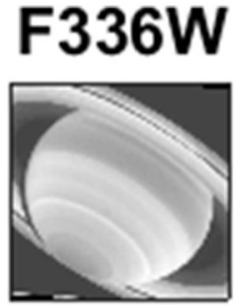

18:04
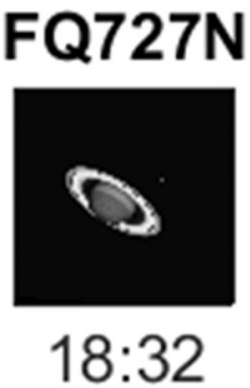
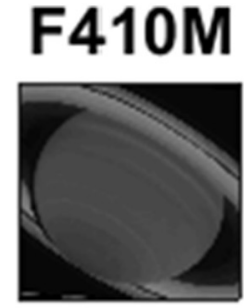

$18: 39$
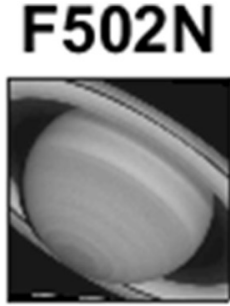

$17: 54$
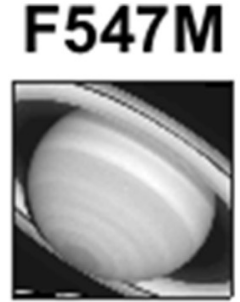

$18: 38$

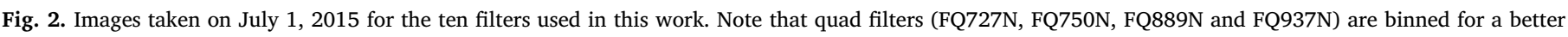
signal-to-noise ratio. 
Table 1

Observations: $B$ (sub-earth planetocentric latitude); $B^{\prime}$ (sub-solar planetocentric latitude); $\alpha$ (phase angle).

\begin{tabular}{|c|c|c|c|c|}
\hline Date & $B$ & $B^{\prime}$ & $\alpha$ & Filters \\
\hline $2015 / 06 / 29$ & 28.74 & 29.62 & 3.65 & F225W, F336W, F410M, F502N, F547M, F689M, FQ727N, FQ750N, FQ889N, FQ937N \\
\hline $2015 / 06 / 30$ & 28.63 & 29.62 & 3.72 & FF225W, F336W, F410M, FF502N, F547M, F689M, FFQ727N, FQ750N, FQ889N, FFQ937N \\
\hline 2015/07/01 & 28.73 & 29.63 & 3.79 & F225W, F336W, F410M, F502N, F547M, F689M, FQ727N, FQ750N, FQ889N, FQ937N \\
\hline
\end{tabular}

a)

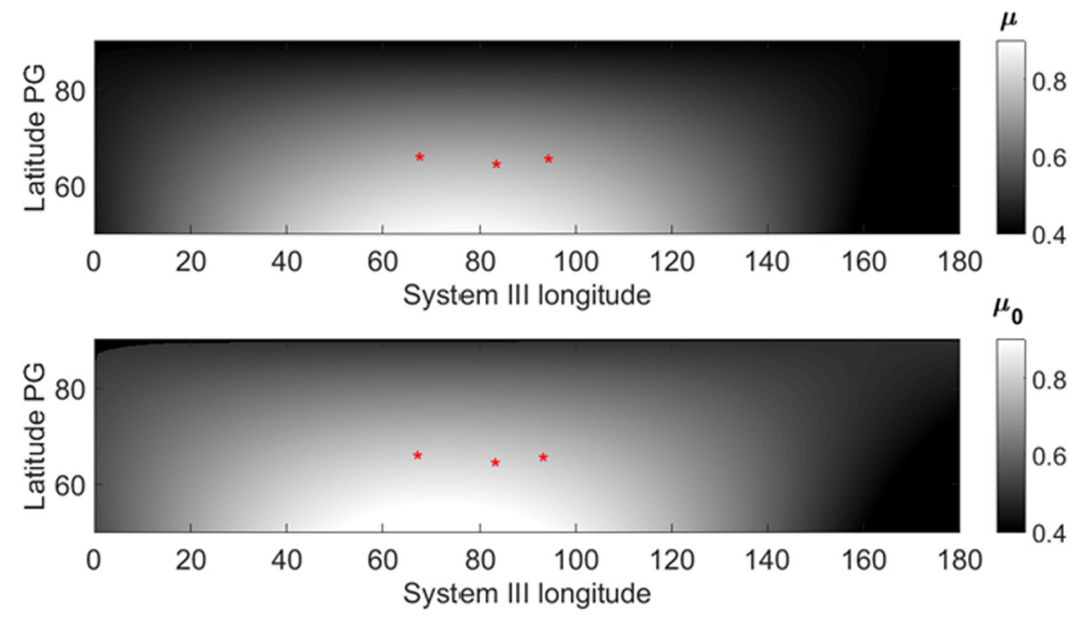

b)
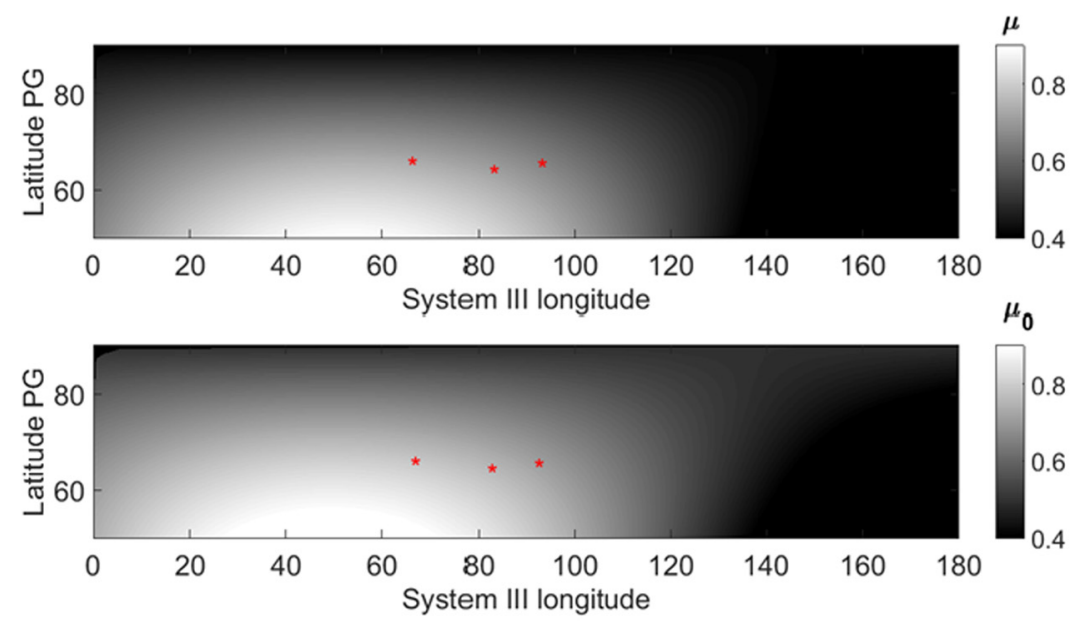

c)

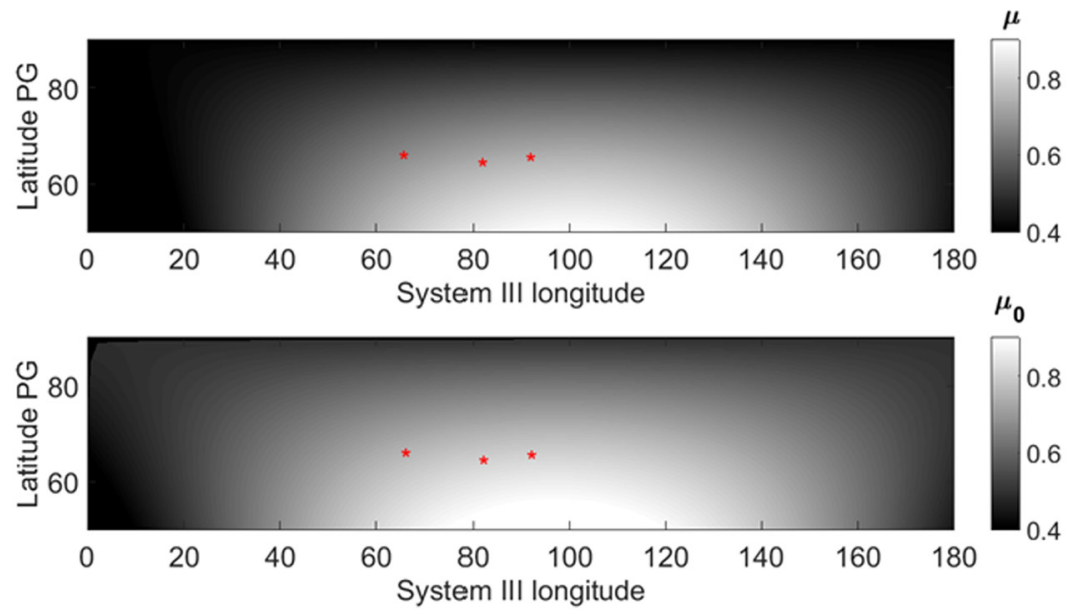

Fig. 3. Maps for the values of the cosine of the emission $(\mu)$ and emission $\left(\mu_{0}\right)$ angles for the different dates of the images used in this work. a) June-29, b) June-30 c) July-1. The location of the triple vortex is indicated on each map with red stars. (For interpretation of the references to color in this figure legend, the reader is referred to the web version of this article.) 

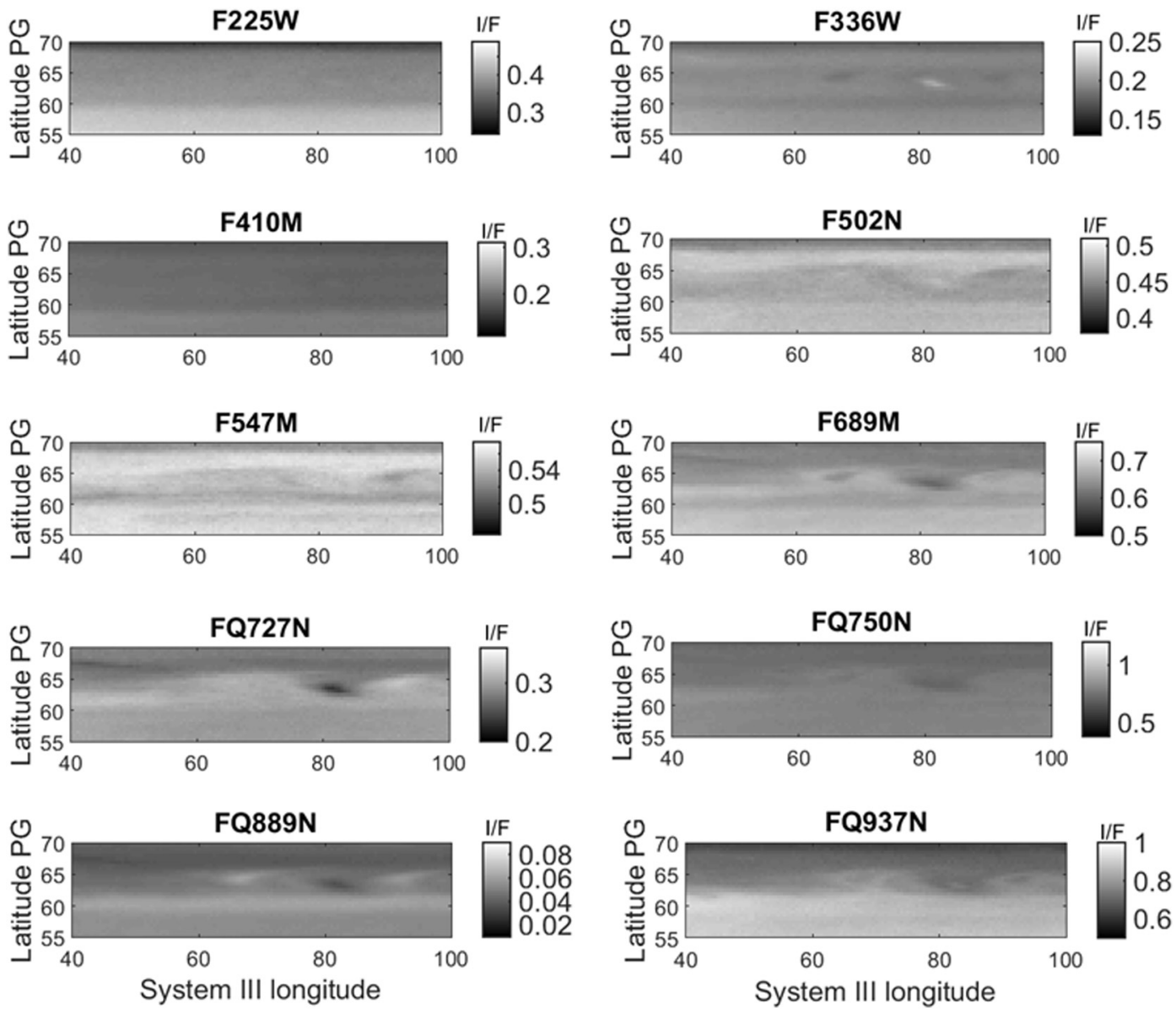

Fig. 4. Cylindrical maps at the available wavelengths showing the region under study with A-C-A system included corrected for limb-darkening. The position of the triple vortex is centered at approximately $85^{\circ} \mathrm{E}$ and is most apparent in filters F336W, FQ889N and FQ937N.

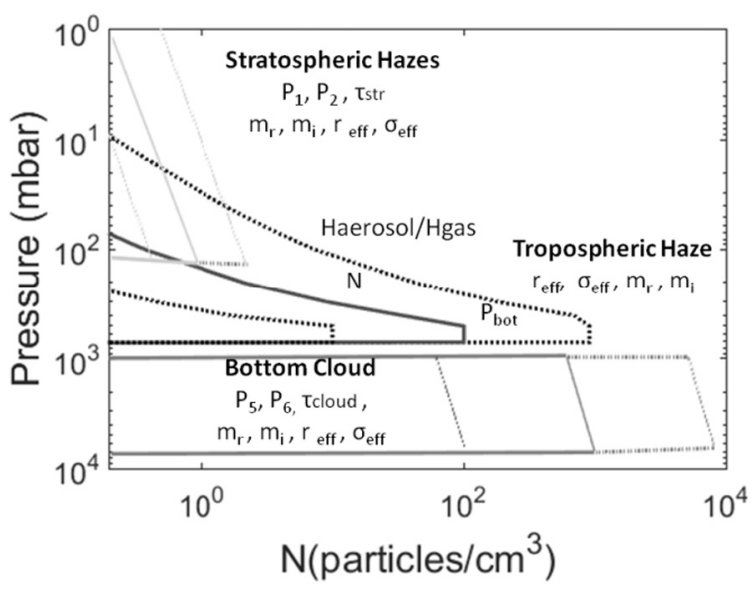

Fig. 5. A priori assumed particle density profiles in our three assumed cloud/ haze layers (solid lines) and their corresponding uncertainties (dotted lines). Parameters of the model for each layer are also indicated (see the text for a full explanation).

Adapted from Sanz-Requena et al. (2018).

F547M, F689M, FQ727N, FQ750N, FQ889N, FQ937N (where the 3digit numbers in the filter name refer to each filter's effective wavelength) and the pixel scale of the images $(300 \mathrm{~km} /$ pixel without binning, proportionally increased in quad filters for optimizing signal to noise and exposure time) are described in Dressel (2019). The ultraviolet filters are, generally speaking, sensitive to Rayleigh scattering by the atmospheric gas and to the properties of the sub-micron sized particles at the upper atmosphere. On the other hand, narrow filters covering methane absorption (FQ727N - intermediate, FQ889N deep), when used together with near continua (FQ750N, FQ937N) are able to provide an altimetry of the cloud tops. A first estimation of the relative altitudes of the triple vortex, with the anticyclones bright at methane bands and dark at short wavelengths, and the opposite for the cyclone, provides a crude picture of the two anticyclones located higher than the cyclone. A similar behavior is observed in images taken with the Imaging Science Subsystem (ISS) onboard the Cassini spacecraft (del Río-Gaztelurrutia et al., 2010; Vasavada et al., 2006) where cyclones appear bright in the BL1 filter and dark in the MT2 and MT3 filters. However, in order to retrieve a more detailed description of the vertical structure, we need to perform a detailed radiative transfer analysis of the data.

\subsection{Data selection}

From all the available data, we have selected a range of latitudes, from $55^{\circ} \mathrm{N}$ to $69^{\circ} \mathrm{N}$ where we can observe the atmospheric feature of interest and the structure of bands and zones in the surrounding background atmosphere.

Since we have images taken on different days, there is a longitude drift in the positions of individual features (such as the vortices) following the zonal wind profile (García-Melendo et al., 2011). We have considered such a drift, and studied the longitude box surrounding the triple vortex that is visible at the three visits, at least in one image for each of them. When more than one observation was available, the values of reflectivity and geometry were averaged, since the differences in observing conditions were small for each visit. This allowed us to obtain 
Table 2

Model atmosphere parameters. Please note that $\tau_{\text {trop }}$ is in fact computed from the other parameters describing the vertical distribution.

\begin{tabular}{|c|c|c|c|}
\hline Layer & Parameter & Type & Value \\
\hline \multirow[t]{7}{*}{ Stratospheric haze } & $\mathrm{P}_{1}$ & Fixed & $1 \mathrm{mbar}$ \\
\hline & $\mathrm{P}_{2}$ & Fixed & 100 mbar \\
\hline & $\tau_{\mathrm{str}}$ & Free & $0.01 \pm 0.01$ \\
\hline & $\mathrm{m}_{\mathrm{r}}$ & Fixed & Amonnia ice \\
\hline & $\mathrm{m}_{\mathrm{i}}$ & Fixed & Amonnia ice \\
\hline & $\mathrm{r}_{\text {eff }}$ & Fixed & $0.1 \mu \mathrm{m}$ \\
\hline & $\sigma_{\text {eff }}$ & Fixed & $0.1 \mu \mathrm{m}$ \\
\hline \multirow[t]{17}{*}{ Tropospheric haze } & $\mathrm{P}_{\text {bot }}$ & Free & $600 \pm 100 \mathrm{mbar}$ \\
\hline & $\mathrm{N}$ & Free & $20 \pm 10$ particles $/ \mathrm{cm} 3$ \\
\hline & $\mathrm{H}$ & Free & $25 \pm 5 \mathrm{~km}$ \\
\hline & $\tau_{\text {trop }}$ & Computed & $10 \pm 5$ \\
\hline & $\mathrm{r}_{\text {eff }}$ & Free & $1.5 \pm 0.5 \mu \mathrm{m}$ \\
\hline & $\sigma_{\text {eff }}$ & Free & $0.1 \pm 0.1 \mu \mathrm{m}$ \\
\hline & $\mathrm{m}_{\mathrm{r}}$ & Fixed & 1.43 \\
\hline & $\mathrm{m}_{\mathrm{i}}(225 \mu \mathrm{m})$ & Free & $10^{-3} \pm 10^{-3}$ \\
\hline & $\mathrm{m}_{\mathrm{i}}(336 \mu \mathrm{m})$ & Free & $10^{-3} \pm 10^{-3}$ \\
\hline & $\mathrm{m}_{\mathrm{i}}(410 \mu \mathrm{m})$ & Free & $10^{-3} \pm 10^{-3}$ \\
\hline & $\mathrm{m}_{\mathrm{i}}(502 \mu \mathrm{m})$ & Free & $10^{-3} \pm 10^{-3}$ \\
\hline & $\mathrm{m}_{\mathrm{i}}(547 \mu \mathrm{m})$ & Free & $10^{-3} \pm 10^{-3}$ \\
\hline & $\mathrm{m}_{\mathrm{i}}(689 \mu \mathrm{m})$ & Free & $10^{-3} \pm 10^{-3}$ \\
\hline & $\mathrm{m}_{\mathrm{i}}(727 \mu \mathrm{m})$ & Free & $10^{-3} \pm 10^{-3}$ \\
\hline & $\mathrm{m}_{\mathrm{i}}(750 \mu \mathrm{m})$ & Free & $10^{-3} \pm 10^{-3}$ \\
\hline & $\mathrm{m}_{\mathrm{i}}(889 \mu \mathrm{m})$ & Free & $10^{-3} \pm 10^{-3}$ \\
\hline & $\mathrm{m}_{\mathrm{i}}(937 \mu \mathrm{m})$ & Free & $10^{-3} \pm 10^{-3}$ \\
\hline \multirow{7}{*}{ Bottom cloud } & $P_{5}$ & Fixed & $1.0 \mathrm{bar}$ \\
\hline & $\mathrm{P}_{6}$ & Fixed & 1.4 bar \\
\hline & $\tau_{\text {cloud }}$ & Free & $10 \pm 5$ \\
\hline & $\mathrm{m}_{\mathrm{r}}$ & Fixed & 1.43 \\
\hline & $\mathrm{m}_{\mathrm{i}}$ & Fixed & $10^{-3}$ \\
\hline & $r_{\text {eff }}$ & Fixed & $10 \mu \mathrm{m}$ \\
\hline & $\sigma_{\text {eff }}$ & Fixed & $0.1 \mu \mathrm{m}$ \\
\hline
\end{tabular}

three spectra for every point of the region of interest, each one at a different viewing and illumination conditions (Fig. 3).

In Fig. 4 we show cylindrical projections of the region of interest in every filter. These images have been corrected for limb-darkening only for display purposes, as the limb-darkening information will be used in the following sections to constrain atmospheric properties. These images are averages from one or more original images, depending on the latitude and longitude coverage of the HST observations for each case.

\section{Methodology}

\subsection{Radiative transfer code}

Our goal is to reproduce the observed dependence of absolute reflectivity with geometry (three combinations of incidence and emission angles) for all wavelengths at the same time, so we can deduce the values of different parameters that give us information about the atmosphere such as the optical thicknesses of aerosol layers, the mean size of the particles, the height at which they are found the different layers and so on. To do this we used the radiative transfer code and retrieval suite NEMESIS, developed by Irwin et al. (2008). This code uses the optimal estimator scheme to find the most likely model that best accounts for the observations.

The version of the code used here is based in a doubling-adding scheme that assumes a plane-parallel atmosphere to compute the emergent intensity of reflected sunlight due to scattering and absorption from atmospheric aerosols and gases. In our model we also take into account the Rayleigh scattering due to the mixture of $\mathrm{H}_{2}$ and $\mathrm{He}$, as well as the absorption due to $\mathrm{CH}_{4}$. The general assumptions (temperature-pressure profile and gaseous abundances) used in this work are the same as in Sanz-Requena et al. (2018).

\subsection{Vertical cloud structure model}

Previous works (Pérez-Hoyos et al., 2005; Sanz-Requena et al., 2018) have found that a vertical structure consisting of three distinct layers of particles is good enough to reproduce the spectral and geometric variations of the absolute reflectivity at visible wavelengths. The overall vertical distribution of particles assumed in the present work is similar to that of Sanz-Requena et al., 2018, as shown in Fig. 5. In Table 2, we summarize the list of free and fixed parameters, which have been chosen according to previous works (Pérez-Hoyos et al., 2016; Sanz-Requena et al., 2018). The same is true for the description of the gaseous scattering (by a mixture of $\mathrm{H}_{2}$ and $\mathrm{He}$, with a volume mixing ratio of 0.124 relative to $\mathrm{H}_{2}$ ), (De Pater and Lissauer, 2001) and absorption. We only considered absorption by $\mathrm{CH}_{4}$, using pre-computed ktables based on the absorption coefficients given by Karkoschka and Tomasko (2010).

The uppermost aerosol layer corresponds to the stratospheric haze that is located between $P_{1}=1 \mathrm{mbar}$ and $P_{2}=100 \mathrm{mbar}$ (Pérez-Hoyos et al., 2005). In this layer, we assume a constant refractive index with real part and imaginary parts for all wavelengths, which we set to the average of that for ammonia ice $\left(m_{r}=1.43\right.$ and $m_{i}=10^{-3}$; PérezHoyos et al., 2016). We also set the effective radius and the effective variance to be $0.1 \mu \mathrm{m}$ and 0.1 , respectively, and assume that the particle size distribution follows a log-normal distribution (Hansen and Travis, 1974). The only free parameter in this layer is the optical thickness, for which we have set a starting point $\tau_{\text {str }}=0.01 \pm 0.01$ (Sanz-Requena et al., 2018), at $900 \mathrm{~nm}$, which will be used as the reference wavelength in the following analysis, except where stated otherwise.

The second layer, corresponding to the tropospheric haze, is characterized by a variable optical thickness $\left(\tau_{\text {trop }}=10 \pm 2\right)$ (Karkoschka and Tomasko, 2005), as well as a parameterization of its vertical distribution. This is defined by the pressure corresponding to the lower base (600 \pm 100 mbar; Fletcher et al., 2007, Roman et al., 2013). The particle-to-gas scale height ratio of this aerosol layer is taken initially as $\mathrm{H}_{\text {aerosol }} / \mathrm{H}_{\mathrm{g}}=0.7 \pm 0.1$ (Pérez-Hoyos et al., 2016) and the value of the initial maximum concentration of particles is $\mathrm{N}=20 \pm 10$ particles/ $\mathrm{cm}^{3}$ (Sanz-Requena et al., 2018). Here $\mathrm{H}_{\mathrm{g}}$ is the atmospheric (gas) scale height $\sim 38 \mathrm{~km}$.

We assume that the particles are spherical and we use Mie theory to compute the phase function. Since all the observations are made at a similarly low phase angle value $\left(\sim 3.6^{\circ}\right)$, this assumption is not critical. We have taken the initial values of $r_{\text {eff }}=1.5 \pm 0.5$ and $\sigma_{\text {eff }}=0.1 \pm 0.1$ (Ortiz et al., 1996). Our model calculates the real refractive index from Kramers-Kronig's relation (Lucarini et al., 2005) from an initial value $m_{r}=1.43$. The imaginary refractive index is set as a free parameter taking as initial value $m_{i}=10^{-3} \pm 10^{-3}$ for all wavelengths (Roman et al., 2013).

The lower layer is fixed between pressures $P_{5}=1.0 \mathrm{bar}$ and $P_{6}=1.4$ bar and corresponds to the cloud putatively formed by ammonia ice (Roman et al., 2013). It must be noted that such ammonia ice has been very rarely spectroscopically identified, with a few exceptions (Baines et al., 2009; Sromovsky et al., 2013). The refractive indices are fixed $\left(m_{r}=1.43\right.$ and $\left.m_{i}=10^{-3}\right)$ as in the stratospheric haze, and the optical thickness is the only free parameter (with a priori values of $\tau_{\text {cloud }}=10 \pm 2$ from Pérez-Hoyos et al., 2016). As in stratospheric haze, the particle size distribution is log-normal with an effective radius of $10 \mu \mathrm{m}$ and an effective variance of $0.1 \mu \mathrm{m}$ (West et al., 2009).

\subsection{Fitting strategy}

To estimate the goodness of fit between the observed and modelled reflectivities, we evaluated the error function $\chi^{2} / n$ at every point of the free-parameter space. The error function is defined at each filter observation as: 


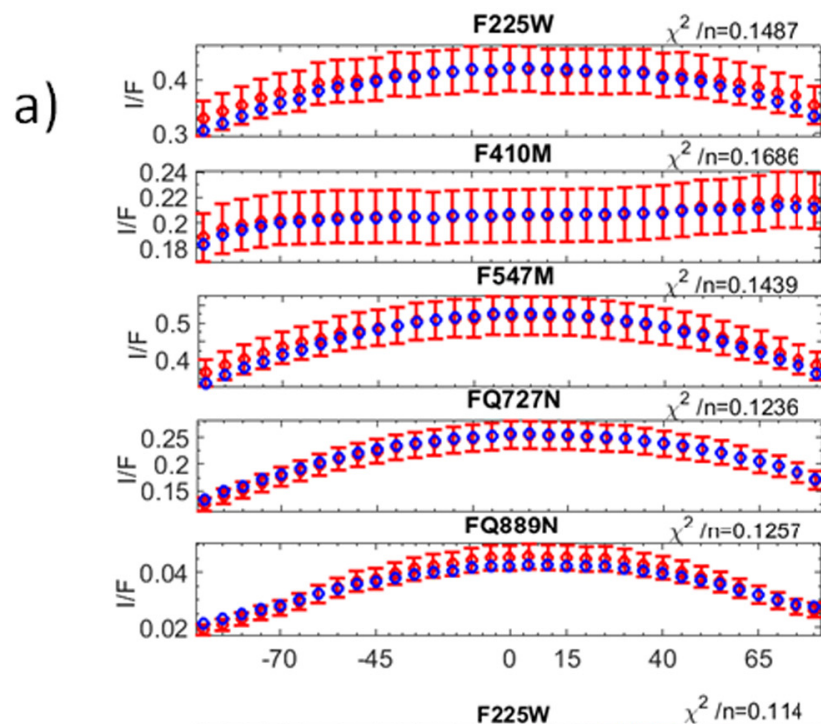

b)

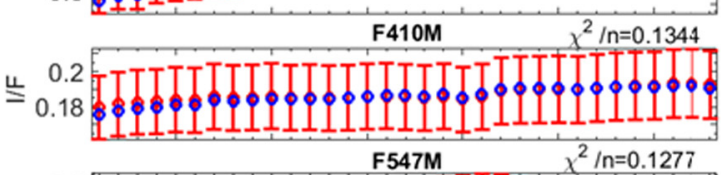

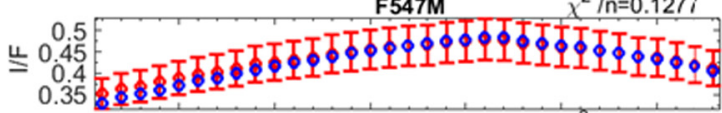

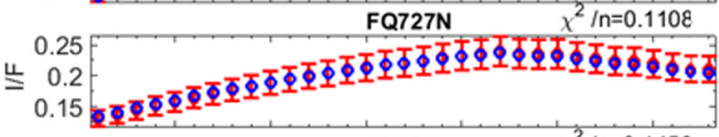

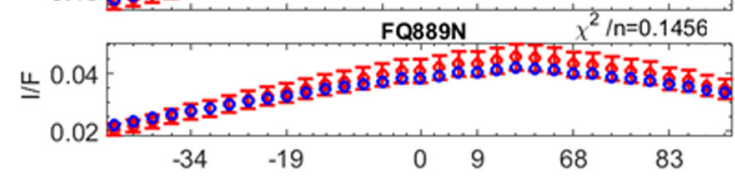
F225W $\quad \chi^{2} / \mathrm{n}=0.1034$

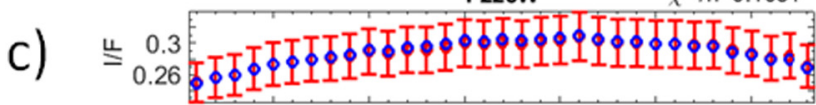

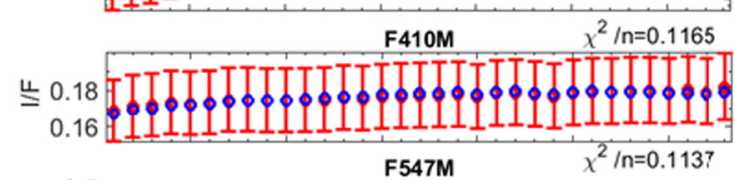

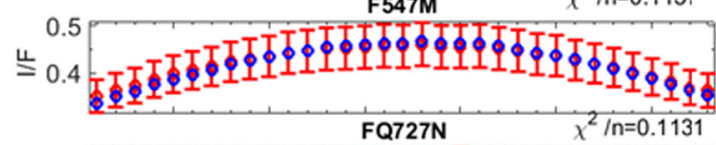

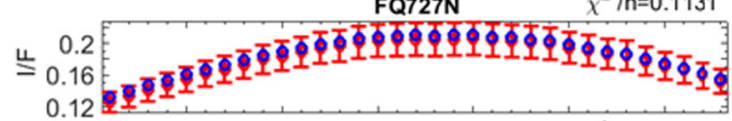

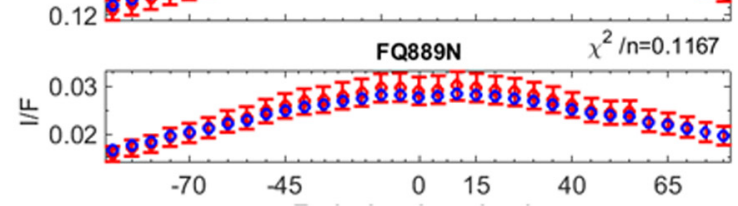

Relative longitude

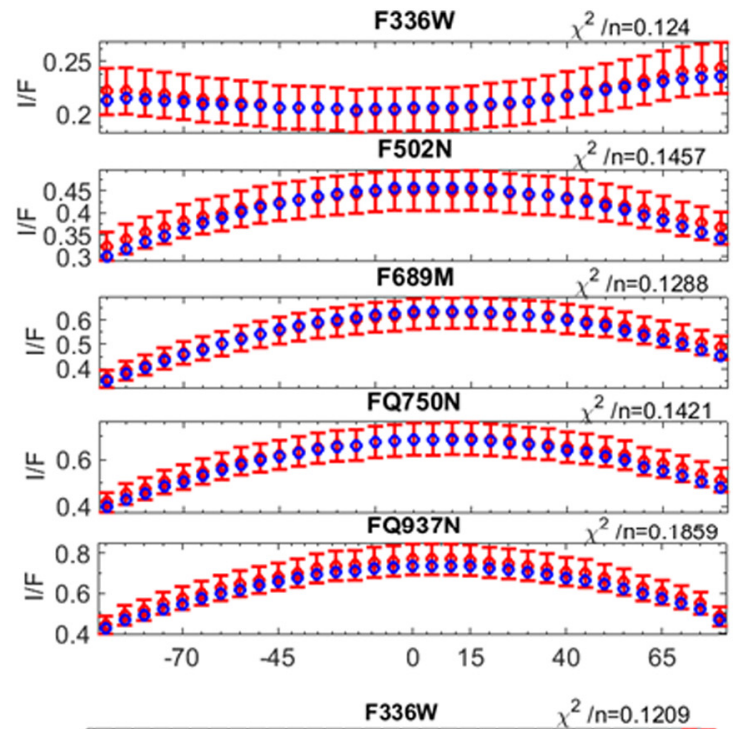

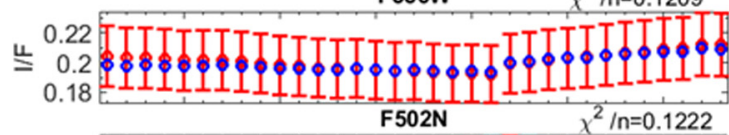

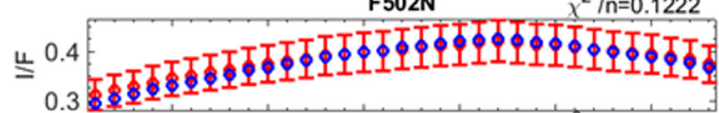

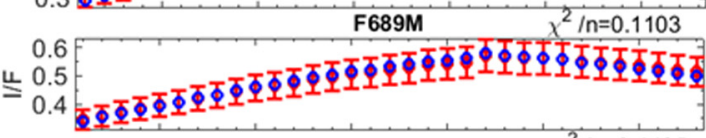

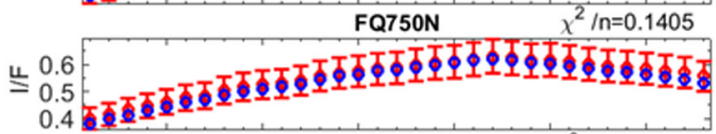

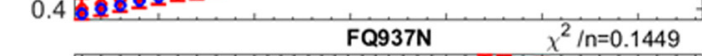

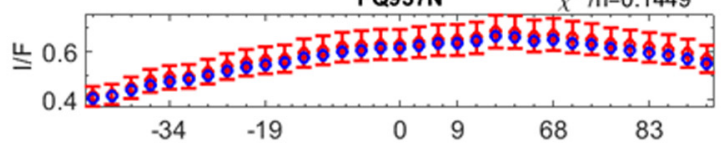
F336W $\quad \chi^{2} / \mathrm{n}=0.1255$

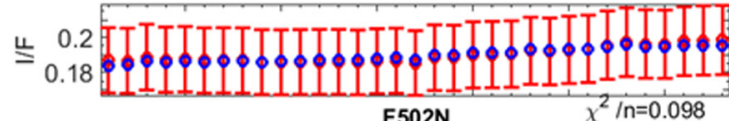

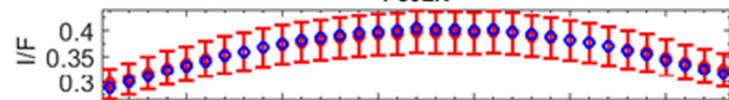

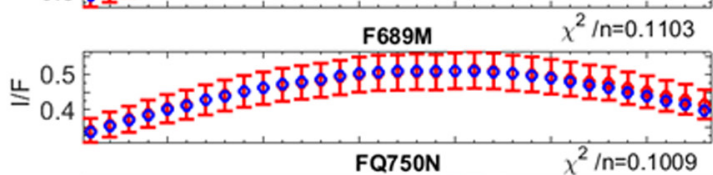

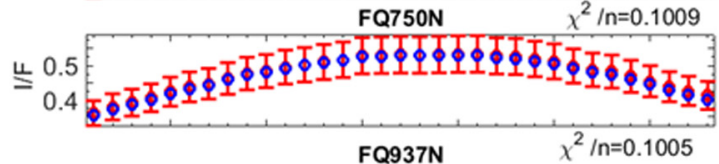

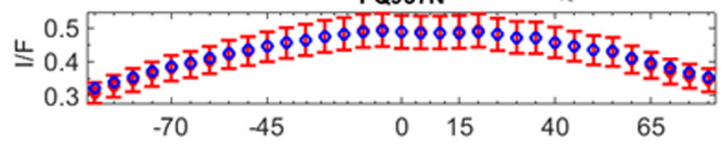
Relative longitude

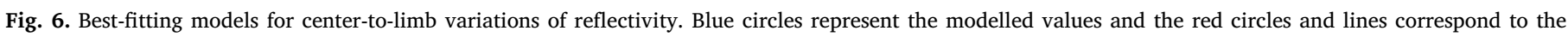

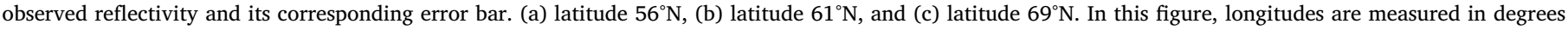

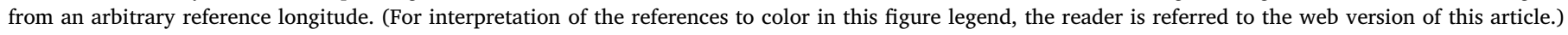


a)

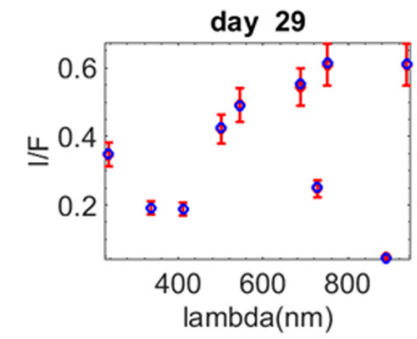

day 29

b)

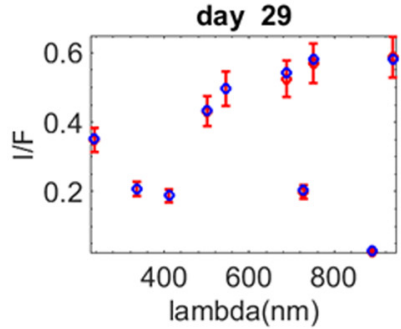

c)

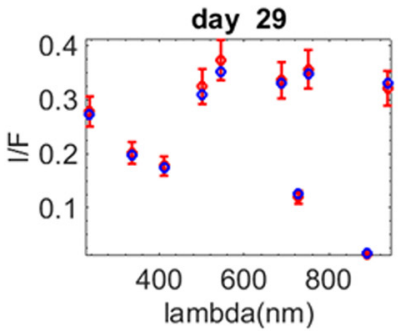

day 29

d)

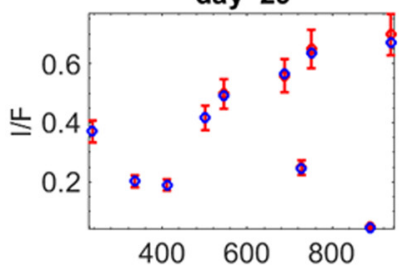

day 29

e)

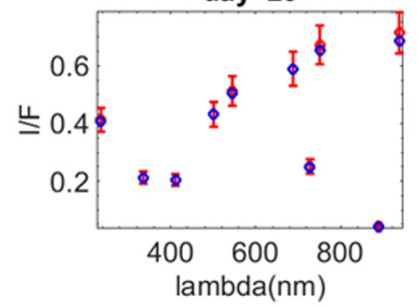

day 30

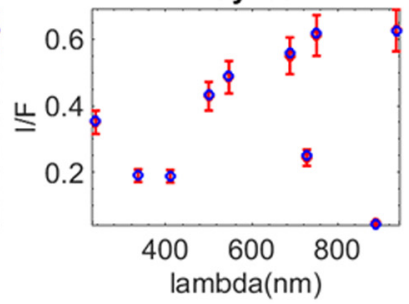

day 30

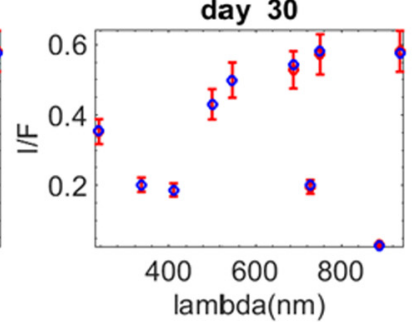

day 30

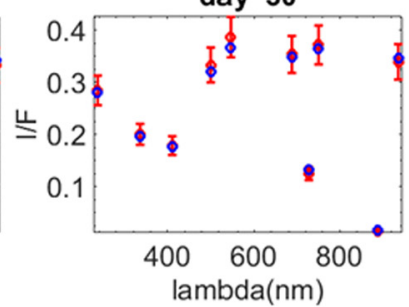

day 30

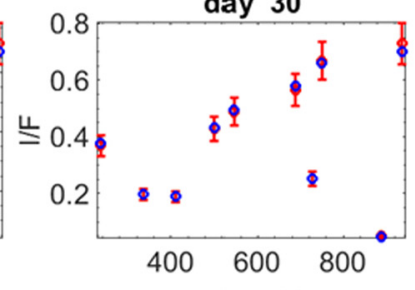

day 30

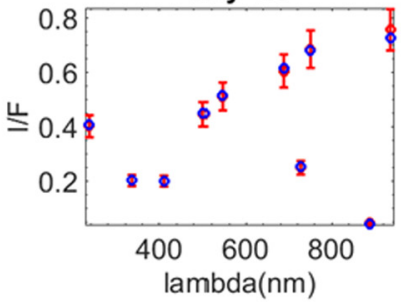

day 1

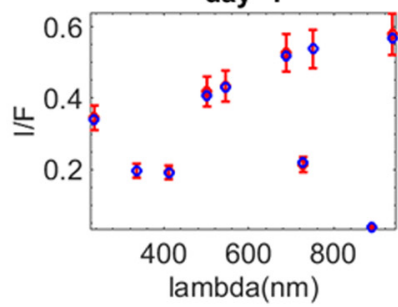

day 1

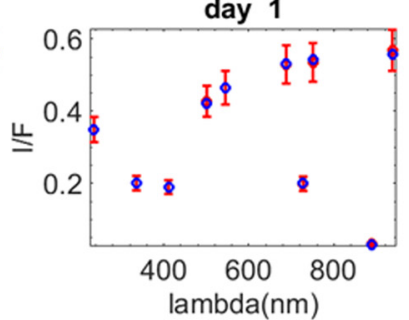

day 1

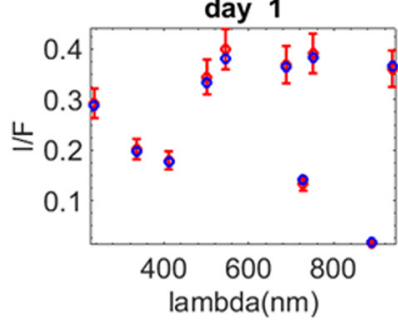

day 1

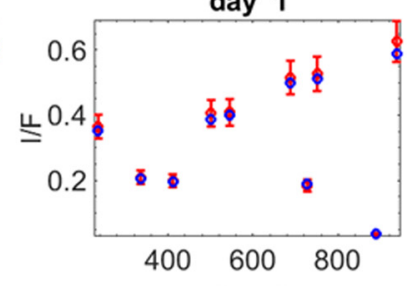

day 1

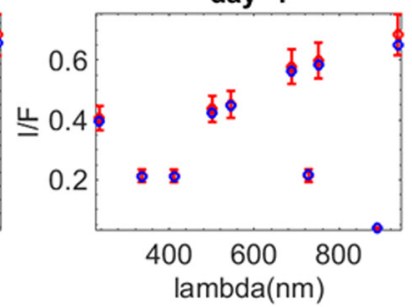

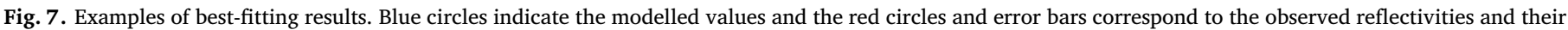

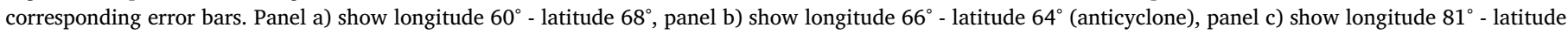

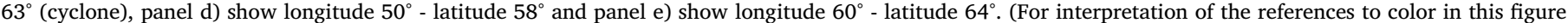
legend, the reader is referred to the web version of this article.)

$\frac{\chi_{\lambda}^{2}}{n}=\frac{1}{n} \sum_{i=1}^{n} \frac{1}{\sigma_{i}^{2}}\left[(I / F)_{o b s}-(I / F)_{m o d}\right]^{2}$

where $n$ is the number of points to be fitted by the model (e.g., the number of points scanning over longitude at a given latitude); $\sigma_{i}$ is the error in the ith measurement; and $(I / F)_{\text {obs }}$ is the observed and $(I / F)_{\bmod }$ the modelled reflectivity at a given point. Then we calculate a reduced $\chi^{2} / n$, an average of all the filters and positions over the disk being modelled. When $\chi^{2} / n$ is larger than one, the profiles deviate systematically from the data. When $\chi^{2} / n$ is smaller than 1 , we can accept the model as it is, on average, inside the data error bars. Our goal is to find models close enough to the data for all geometries and wavelengths $\left(\chi^{2} / n<1\right)$ at every point of the region of interest. This approach does not take into account any differences between systematic and random errors and only minimizes the overall model deviation from the data. As low pixel-to-pixel noise allows determining limb-darkening more precisely than absolute reflectivity values (mostly affected by systematic errors), we will discuss in Section 4.1 how well best-fitting models are able to match the observed limb-darkening.

We first fitted center-to-limb brightness profiles for the range of selected latitudes $55^{\circ}$ to $69^{\circ} \mathrm{N}$ with intervals of $0.5^{\circ}$, excluding the regions where the triple vortex is present. In this way, we obtain a reference model that we use later, including the regions where the triple vortex is located. We initially fitted filters FQ727N, FQ750N, FQ889N and FQ937N with a constant value of $m_{i}=10^{-3}$. Once the result was acceptable we added the shortest wavelengths (filters F225W, F336W, F410M, F502N, F547M and F689M) leaving also the refractive indices as free parameters. This procedure avoids overfitting with the imaginary refractive index (whose values are forced to be nearly flat for longer wavelengths), provides good results and it is very similar to the 

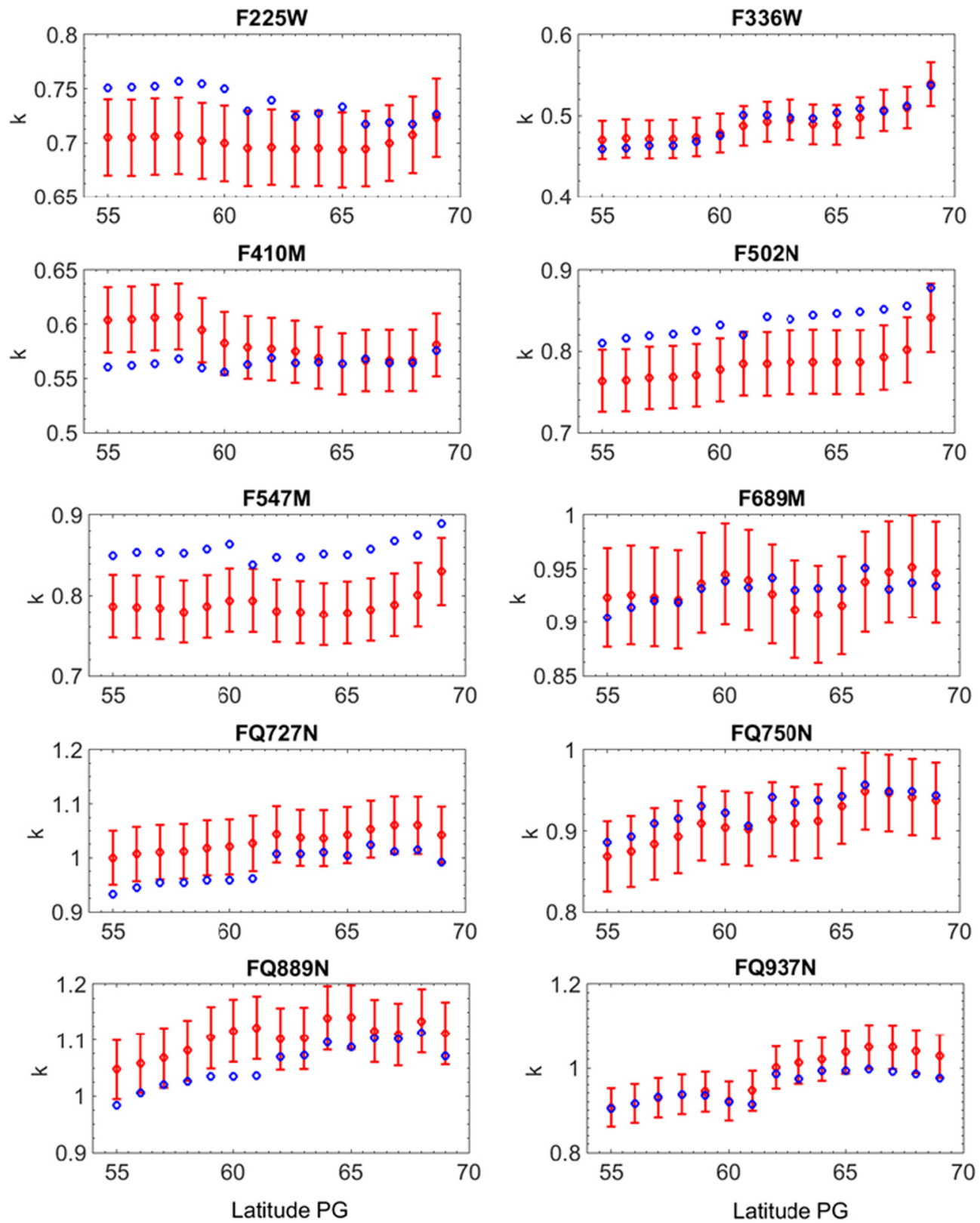

Fig. 8. Values of the limb darkening coefficient $k$ for all latitudes $\left(55^{\circ} \mathrm{N}\right.$ to $69^{\circ} \mathrm{N}$ and filters (F225W, F336W, F410M, F502N, F547M, F689M, FQ727N, FQ750N, FQ889N, FQ937N). Blue circles correspond to the best fits and the red circles and lines correspond to the HST data and their error bar. Error bars for the observed limb-darkening is taken as a $5 \%$ of the value of the HST data. This includes not only pixel-to-pixel random noise but also navigation uncertainties. (For interpretation of the references to color in this figure legend, the reader is referred to the web version of this article.)

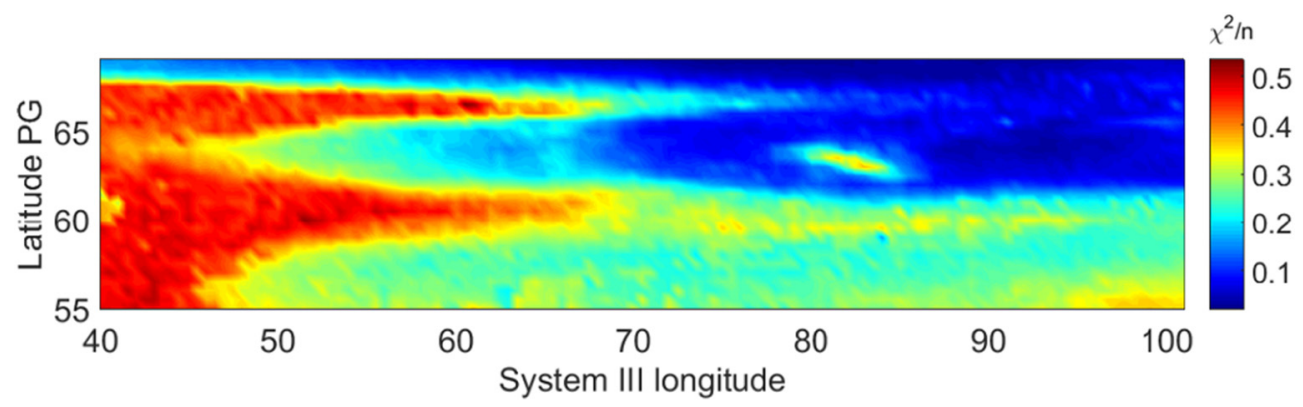

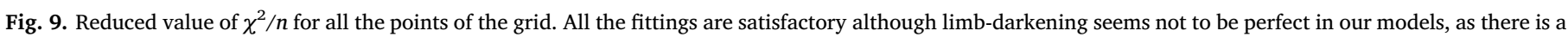
longitude trend in the goodness of fit. 


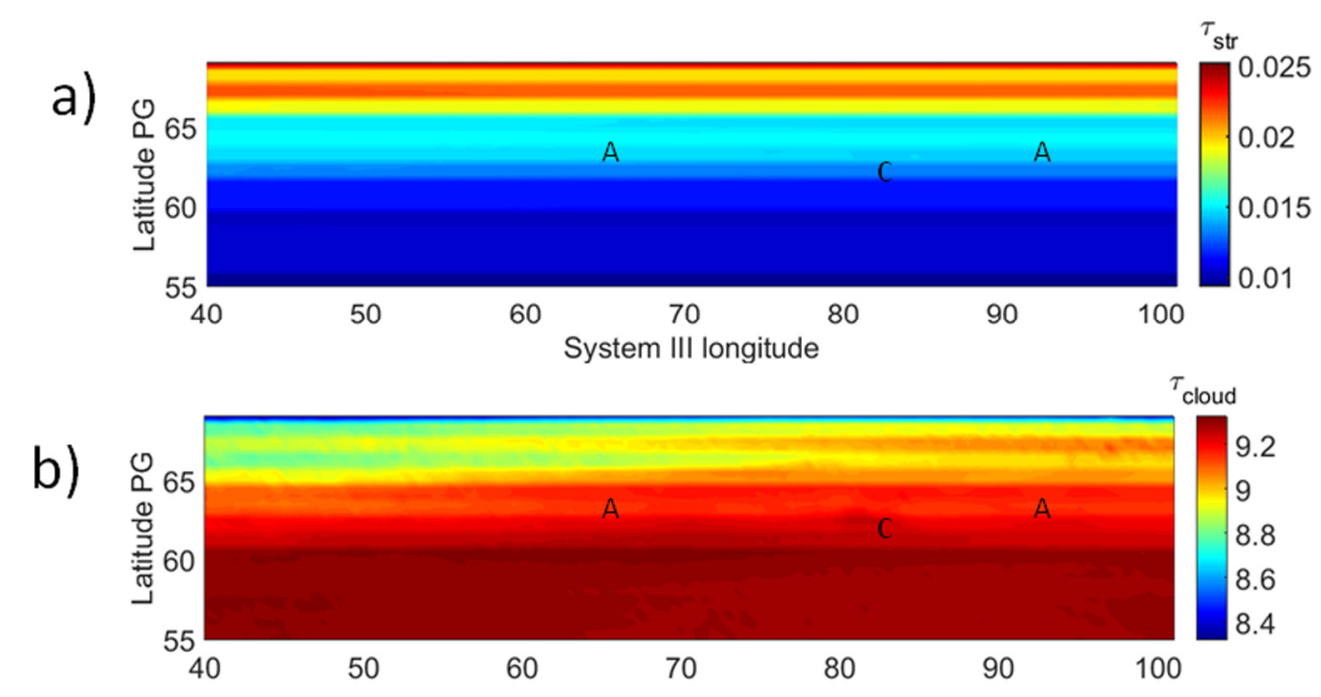

System III longitude

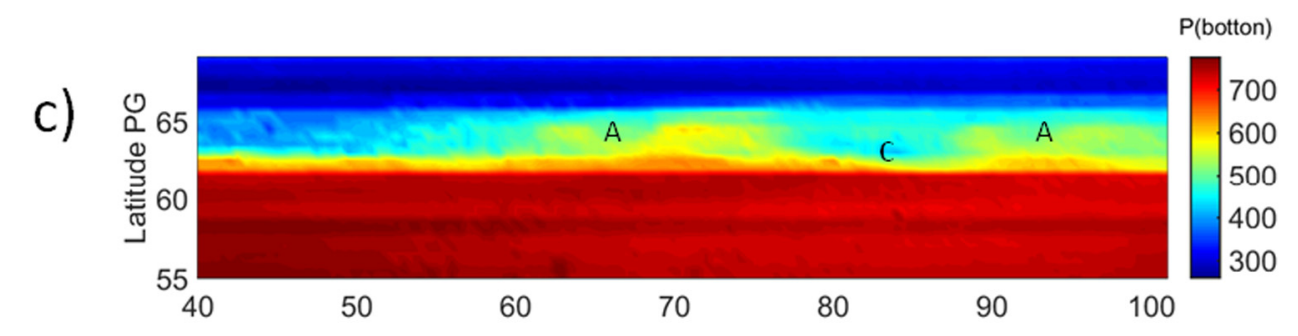

System III longitude

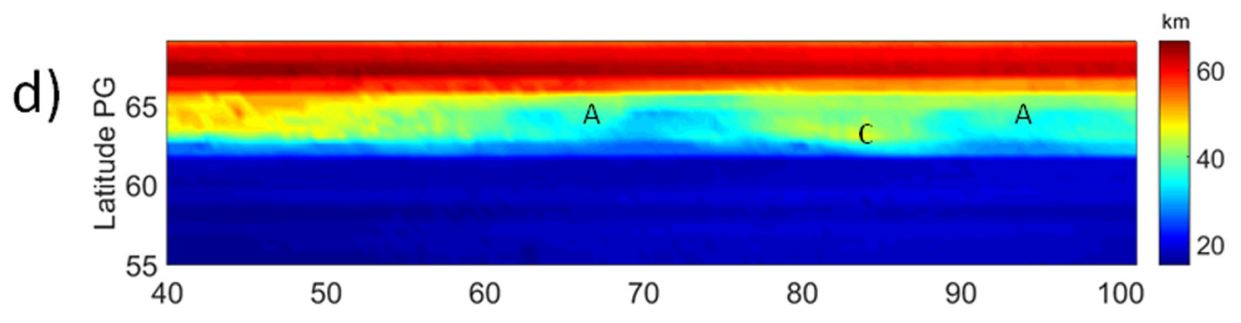

System III longitude

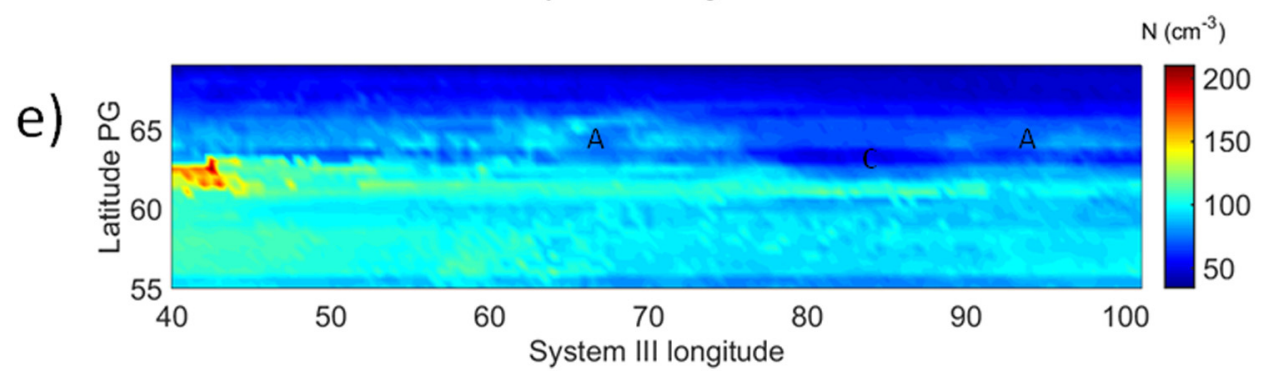

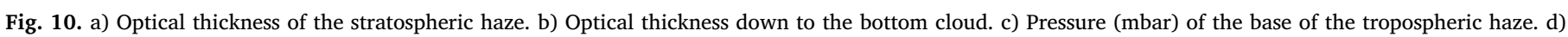

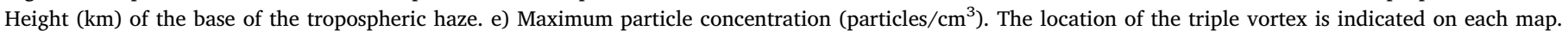
(Anticyclone (A), Cyclone (C), Anticyclone (A)).

strategy in Sanz-Requena et al. (2018). The result of this analysis is presented in Fig. 6, where we show the ten filters covering a wide geometrical range.

Once we have a good model for the overall limb-darkening behavior of the reference atmosphere, we want to fit individually all the points in the region of interest, including particular features such as the vortices. For doing so we run new retrievals using as input the reference atmosphere model at a given latitude. In Fig. 7 we show best-fitting model results for each of the three vortices at some locations of interest.

\section{Modelling results}

\subsection{General results and limb-darkening}

We have assumed error bars for the data that do not separate random from systematic errors. In order to rule out and quantify a possible systematic deviation of the limb-darkening in the different regions and filters and in order to improve our model we fitted both observations and best- fitting models to a Minnaert law. 

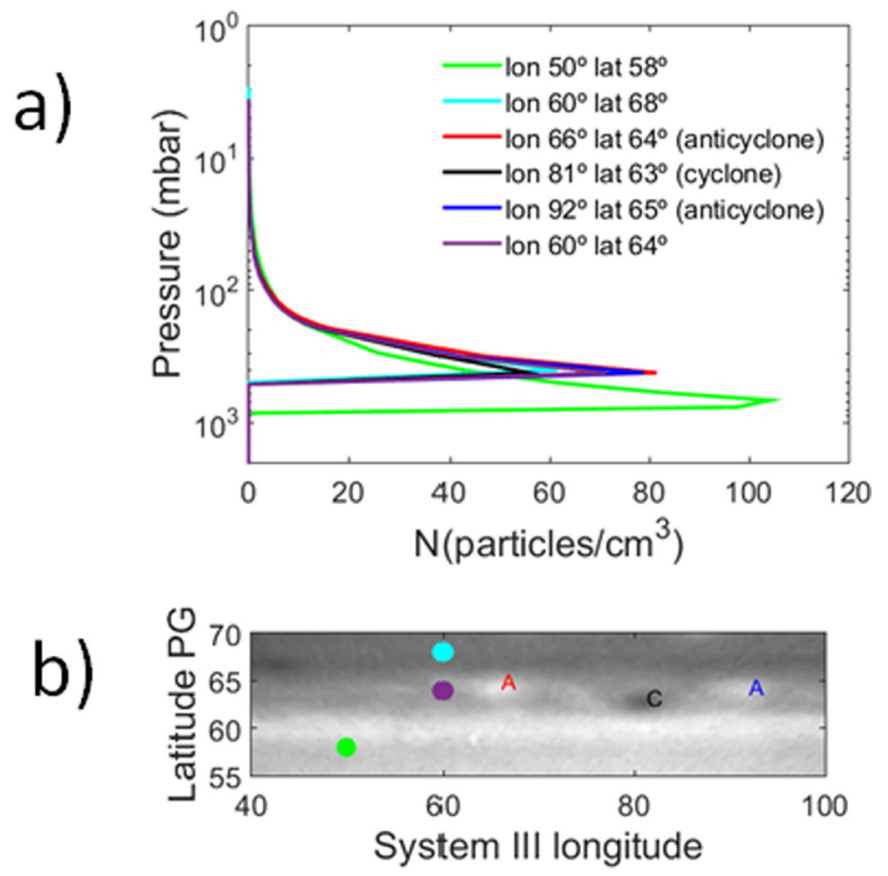

Fig. 11. a) Vertical distribution of the tropospheric particles for the vortices and a reference region. b) Location of vortices and reference regions.

$\frac{I}{F}=\left(\frac{I}{F}\right)_{0} \mu_{0}^{k} \mu^{k-1}$

where $(I / F)_{0}$ is the reference reflectivity value at perfect nadir geometry ( $\mu=1$ and $\mu_{0}=1$ ) and $k$ is a wavelength-dependent limb-darkening coefficient (Sanz-Requena et al., 2018). This analysis corresponds to the background models where center-to-limb data is available, and not to the specific cyclonic features. While previous Fig. 7 explicitly shows the deviation between models and data, we show in Fig. 8 the results corresponding to the variation of the values of $k$ for the different latitudes and for all wavelengths. As previously stated, the limb-darkening can be more precisely determined from the original data, as it is mostly affected by pixel-to-pixel and random errors, which are substantially lower than systematic calibration uncertainties. Although it would be desirable to be able to fit independently the limb-darkening with stronger constraints, our current version of the retrieval code does not have this capability. We want to show here that, at least, our best-fitting models are close enough to the measured limb-darkening values.

There is no systematic deviation in the limb-darkening of the models from that of the data, as the same filter or region can give differences as low as $0.15 \%$ or as high as $7 \%$, with average differences of $3 \%$. Taking into account not only the errors in relative photometry but also the navigation uncertainties (including the longitudinal drift due to zonal winds) the level of discrepancy between observations and models is acceptable and indicates that our model atmosphere reproduces well the observed limb-darkening.

\subsection{Best-fitting results}

In Fig. 9 we show the goodness of the fit $\left(\chi^{2} / n\right)$ for all the points of the region of study. We find that at all points $\chi^{2} / n<1$. However, there is a longitude trend in the goodness of fits, which implies that the overall limb-darkening behavior is not perfectly constrained when we study each point of the grid separately. This implies that the state derived from the limb-darkening analysis is not sufficiently well constrained and the optimal results retrieved from a point-by-point basis do not fully reproduce the observed limb-darkening, as we will discuss later.

Figs. 10 to 14 show the best-fitting parameters as a function of latitude and longitude.

The optical thickness (at $0.9 \mu \mathrm{m}$ ) of the stratospheric haze (Fig. 10a) shows no zonal variation and depends mostly on latitude. We find an increase with latitude that varies from $\tau$ str $=0.01 \pm 0.01\left(\sim 55^{\circ} \mathrm{N}\right)$ to $\tau$ str $=0.025 \pm 0.01\left(\sim 69^{\circ} \mathrm{N}\right)$. This implies an increase of a factor 3 in the stratospheric particle density from the lower to the upper latitudes, similar to that presented in Sanz-Requena et al. (2018). It is important to notice that the models do not require differences in the thickness of the upper haze between the anticyclone-cyclone system and the surrounding regions ( $\tau$ str $\sim 0.015 \pm 0.01$ ).

In Fig. 10b we show the optical thickness down to the bottom cloud at the ammonia condensation levels. In this case we find that the values are quite homogeneous, with hardly any spatial variation ( $\tau_{\text {cloud }}$ $\sim 9.2 \pm 2$ to $\sim 55^{\circ} \mathrm{N}$ and $\tau_{\text {cloud }} \sim 8.4 \pm 2$ to $\sim 69^{\circ} \mathrm{N}$ ).

The pressure level for the base of the tropospheric haze (Fig. 10c) varies from $700 \pm 100 \mathrm{mbar}$ for $\sim 55^{\circ} \mathrm{N}$ to $300 \pm 100 \mathrm{mbar}$ for $\sim 69^{\circ} \mathrm{N}$. The values are very homogeneous in longitude. However, there are small differences in the region of the triple vortex. The value in the anticyclones is approximately $550 \pm 100 \mathrm{mbar}$ while in the cyclone region the average value is $500 \pm 100 \mathrm{mbar}$. This implies that in terms of altitude above the $1 \mathrm{bar}$ level, the tropospheric haze is located at $40 \pm 5 \mathrm{~km}$ and $50 \pm 5 \mathrm{~km}$ for the anticyclones and the cyclone respectively. On the other hand, as we approach higher latitudes the height of the base of the tropospheric haze increases from $20 \pm 5 \mathrm{~km}$ at $55^{\circ} \mathrm{N}$ to $60 \pm 5 \mathrm{~km}$ at $70^{\circ} \mathrm{N}$.

The maximum particle concentration (Fig. 10d) follows a latitudinal behavior similar to that of the base pressure of the tropospheric haze. Its value ranges from $100 \pm 10$ particles $/ \mathrm{cm}^{3}$ for $55^{\circ} \mathrm{N}$ to $50 \pm 10$ particles $/ \mathrm{cm}^{3}$ for $70^{\circ} \mathrm{N}$. Again, the behavior is quite homogeneous in longitude, with small differences at the anticyclones ( $80 \pm 10$ particles $\left./ \mathrm{cm}^{3}\right)$ and the cyclone $\left(55 \pm 10\right.$ particles $\left./ \mathrm{cm}^{3}\right)$, while in the cyclone we can see a decrease in the maximum average concentration.

In Fig. 11a we show the variation of particle density with height for 6 different regions. We observe that for low latitudes $\left(58^{\circ} \mathrm{N}\right)$ maximum concentrations $\left(\sim 110 \pm 10\right.$ particles $\left./ \mathrm{cm}^{3}\right)$ are located at pressures of $\sim 900 \pm 100$ mbar. The maximum concentrations in the two anticyclonic regions $\left(64^{\circ} \mathrm{N}\right.$ and $\left.65^{\circ} \mathrm{N}\right)$ have similar values $(\sim 85 \pm 10$ particles $\left./ \mathrm{cm}^{3}\right)$ and both are at the same pressure level $(\sim 550 \pm 100 \mathrm{mbar})$. In the cyclonic region $\left(63^{\circ} \mathrm{N}\right)$ we observe a smaller peak concentration $\left(\sim 55 \pm 10\right.$ particles $\left./ \mathrm{cm}^{3}\right)$ at a lower pressure $(\sim 500 \pm 100 \mathrm{mbar})$. Outside the triple vortex, we find that at the same latitudes the maximum concentration $(\sim 70 \pm 10$ particles/ $\mathrm{cm}^{3}$ and $\sim 600 \pm 100 \mathrm{mbar}$ ) is smaller than that of the anticyclones and bigger than that of the cyclone. The value of the maximum concentration at higher latitudes $\left(68^{\circ} \mathrm{N}\right)$ is $\sim 60 \pm 10$ particles $/ \mathrm{cm}^{3}$ and is located at a pressure similar to that of the cyclone.

Fig. 12a shows the fractional scale height of the tropospheric haze, Haerosol/Hgas. We observe a decrease of this parameter with latitude, $0.65 \pm 0.1$ (Haerosol $\sim 23 \pm 2 \mathrm{~km}$ ) for $\sim 55^{\circ} \mathrm{N}$ and $0.45 \pm 0.1$ (Haerosol $\sim 16 \pm 2 \mathrm{~km}$ ) for $\sim 69^{\circ} \mathrm{N}$. In the latitudes where the triple vortex is found, we do not observe substantial differences between the values corresponding to the anticyclones and the cyclone $(0.55 \pm 0.1)$ (Haerosol $\sim 18 \pm 2 \mathrm{~km}$ ).

The optical thickness of the tropospheric haze is also quite homogeneous in longitude except at the triple vortex (Fig. 12b). In latitude we observe an increase of optical thickness from $\tau$ trop $\sim 28 \pm 2$ at $55^{\circ} \mathrm{N}$ to a maximum $\tau$ trop $\sim 35 \pm 2$ at $61^{\circ} \mathrm{N}$, and then the magnitude decreases down to $\tau$ trop $\sim 10 \pm 2$ at $69^{\circ} \mathrm{N}$. This is consistent with the belt and zone structure of the region. The values of the optical thickness in the anticyclones are $\tau$ trop $\sim 25 \pm 2$, similar to the average at their latitude, while the optical thickness of the cyclone it is $\sim 10 \pm 2$. As we will see, this parameter accounts for most of the spectral and geometrical variation in this data set.

We have calculated the vertical thickness of the tropospheric haze (Fig. 12c) from the height corresponding to the optical thickness equal to 1 down to the base level, as a proxy to the vertical extension of the 
a)

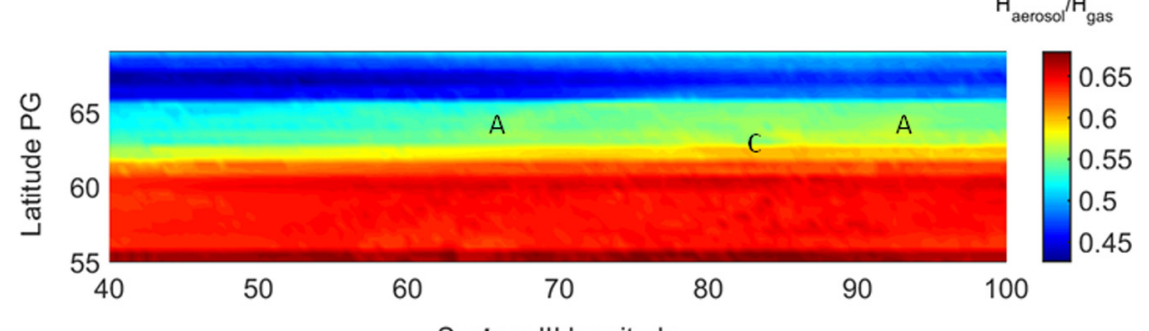

System III longitude

b)

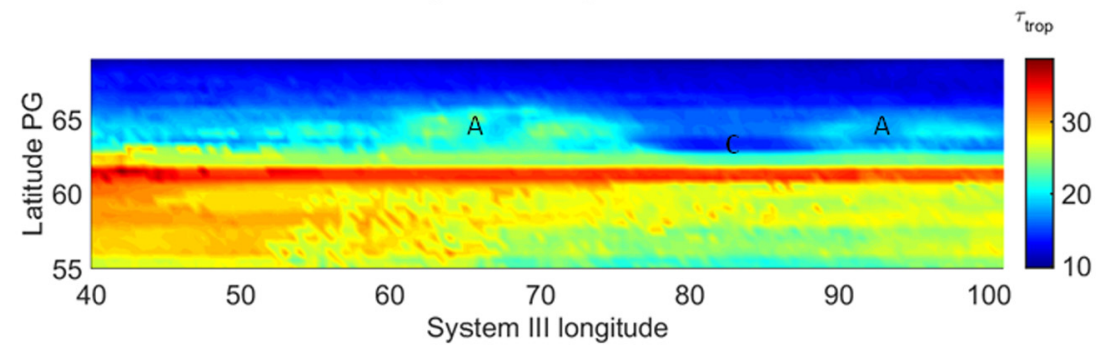

$\tau_{\text {trop }}$

c)

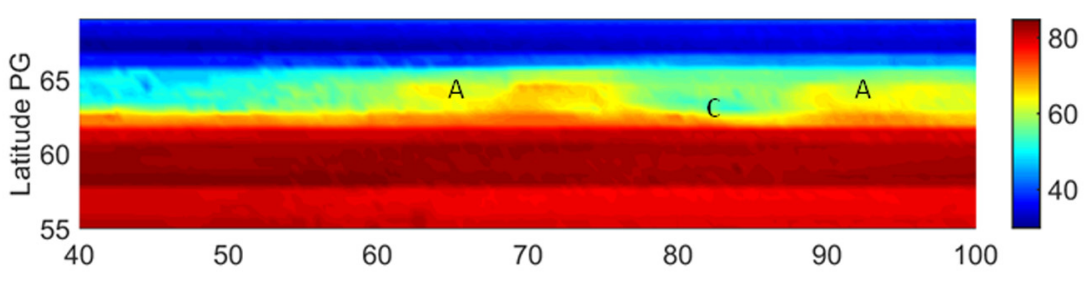

System III longitude

d)

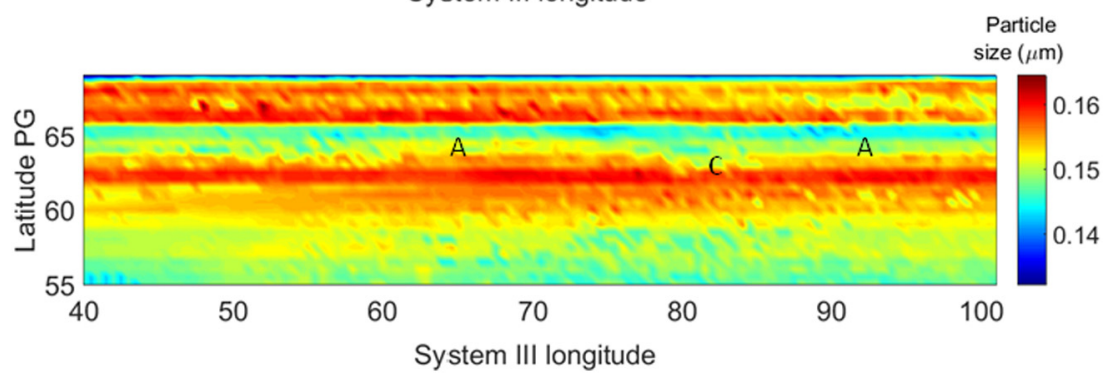

Fig. 12. a) Scale height of the tropospheric haze, $\left(\mathrm{H}_{\text {aerosol }} / \mathrm{H}_{\text {gas }}\right)$. b) Optical thickness of the tropospheric haze. c) Vertical thickness of the tropospheric haze (km). d) Particle size $(\mu \mathrm{m})$. The location of the triple vortex is indicated on each map. (Anticyclone (A), Cyclone (C), Anticyclone (A)).

haze. We observe that the tropospheric haze thickness decreases polewards from $80 \pm 5 \mathrm{~km}$ at $\sim 55^{\circ} \mathrm{N}$ to $40 \pm 5 \mathrm{~km}$ at $\sim 69^{\circ} \mathrm{N}$. Again, the behavior in longitude is quite homogeneous. However, we do find differences between the anticyclonic region and the cyclonic region with thicknesses of $60 \pm 5 \mathrm{~km}$ and $50 \pm 5 \mathrm{~km}$ respectively.

Regarding the particles size, we do not find significant differences for the range of latitudes that we are considering, being $\sim 0.15 \pm 0.1 \mu \mathrm{m}$. (Fig. 12d), with a subtle belt/zone structure that is inside the parameter error bars and thus not statistically significant.

Fig. 13 shows the imaginary refractive indices of the tropospheric haze for six different wavelengths. We observe that the values are quite homogeneous in longitude. We have omited the values for wavelengths $725 \mathrm{~nm}, 750 \mathrm{~nm}, 889 \mathrm{~nm}$ and $937 \mathrm{~nm}$ since they are practically constant. We do not appreciate significant differences at the locations of the anticyclones and the cyclone at any wavelength. The relative errors of all parameters retrievals are displayed in Fig. 14.

In Fig. 15, we show the variation of the imaginary refractive indexes $m_{i}$ with wavelength for six selected regions, including the locations of the cyclone and anticyclones. The behavior at all the six regions is similar. At visible and infrared wavelengths, $m_{i}$ decreases with wavelength, from $\sim 28 \pm 0.110^{-3}(410 \mathrm{~nm})$ to $5 \pm 0.110^{-4}(937 \mathrm{~nm})$. In the shortest wavelengths, it increases from $\sim 7 \pm 0.110^{-3}(225 \mathrm{~nm})$ to $\sim 16 \pm 0.110^{-3}(336 \mathrm{~nm})$ and $\sim 28 \pm 0.110^{-3}(410 \mathrm{~nm})$. In this range of wavelegths, $m_{i}$ is slightly higher at higher latitudes.

Table 3 shows the best-fitting values of the parameters for different regions.

According to our results, the behavior of the parameters is quite homogeneous in longitude both in the stratospheric haze and at cloud level. This same behavior is found in the tropospheric haze, except in the latitudes where the triple vortex appears.

\subsection{Sensitivity to the model parameters}

It is possible to evaluate the information gain during the retrieval process by comparing the relative errors between the a priori assumption and the a posteriori best-fitting value. For doing so, we evaluate the improvement factor as defined by Irwin et al. (2015). A low improvement factor indicates that the a posteriori result of the free parameter is not giving us substantial information regarding the a priori uncertainty, while a high improvement factor indicates that we have significantly reduced the a priori uncertainty during the retrieval. In Table 4 we show the results of the improvement factors for the different free 

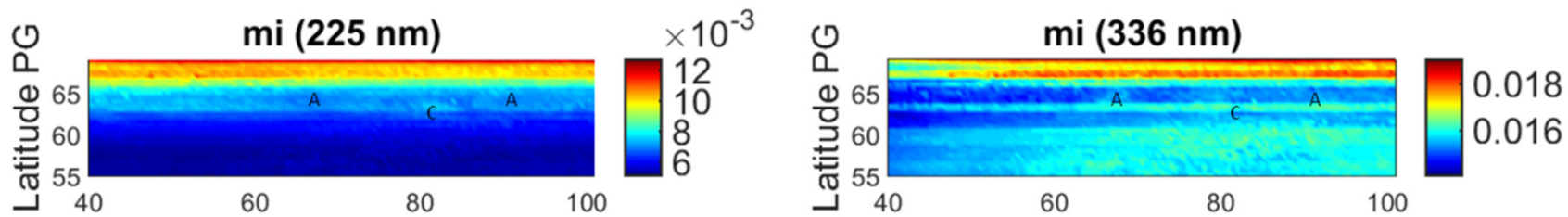

System III longitude

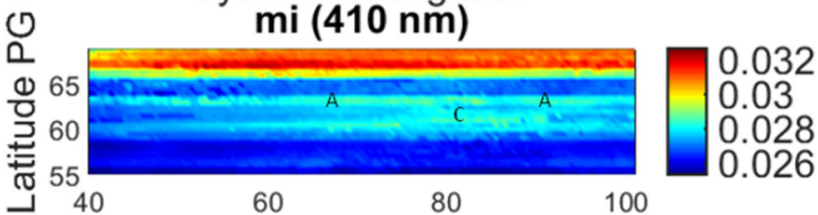

System III longitude

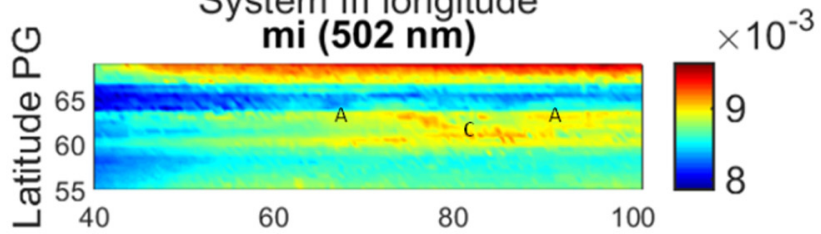

System III longitude
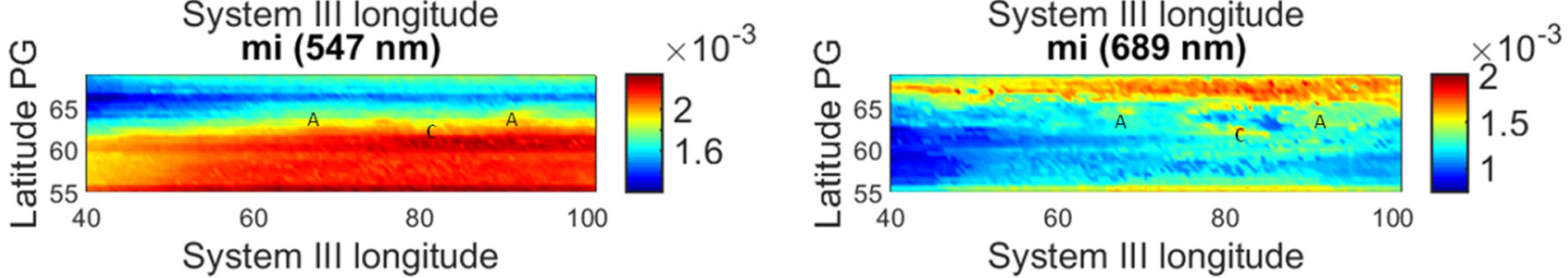

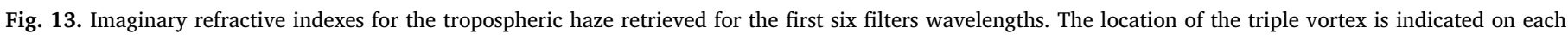
map. (Anticyclone (A), Cyclone (C), Anticyclone (A)).

a)

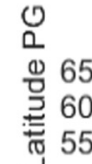

c)

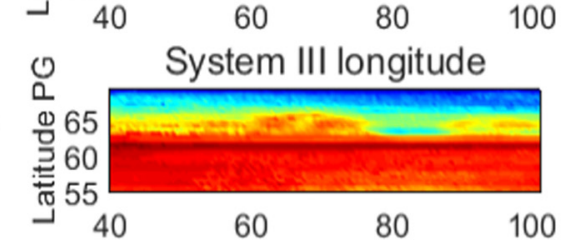

e)

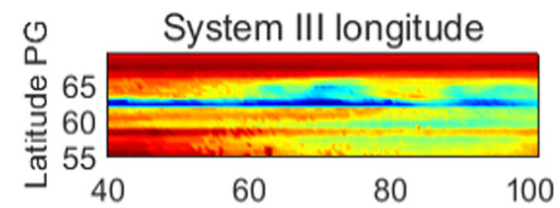

g)

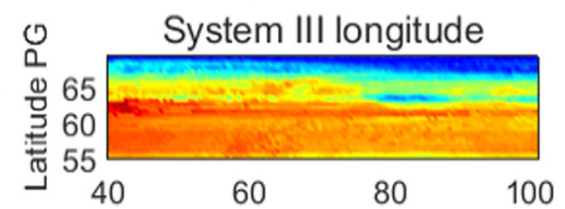

i)

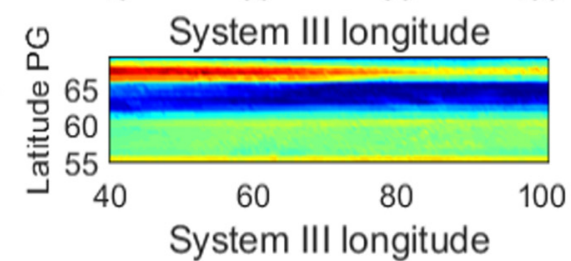

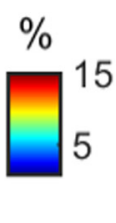
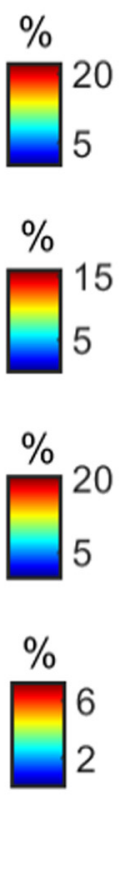

b) $\frac{0}{\square}$

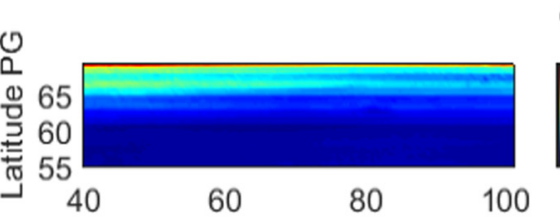

$\%$

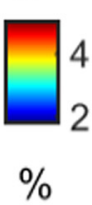

System III longitude

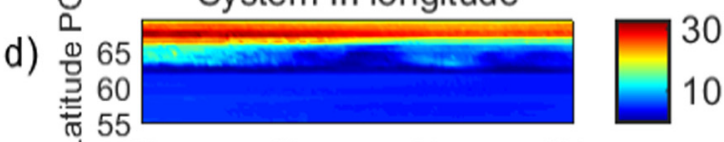

60

80

System III longitude

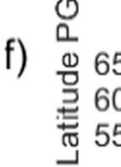

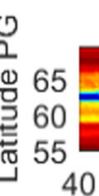

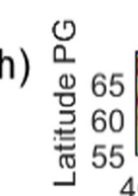

Sys

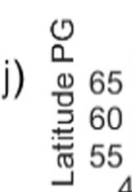

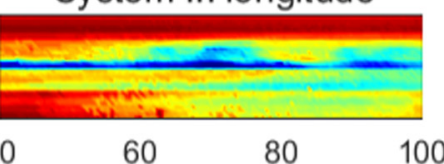

System III longitude

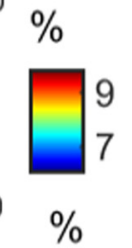

System III longitude

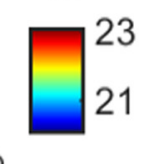

$\%$

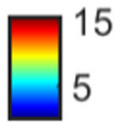

$\%$

$\%$

21

System III longitude

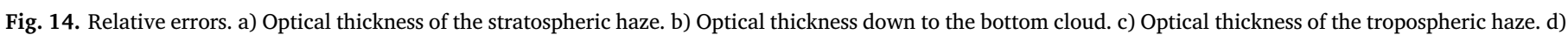

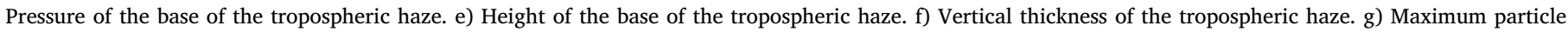
concentration. h) Particle size, i) Scale height of the tropospheric haze, $\left(\mathrm{H}_{\mathrm{aerosol}} / \mathrm{H}_{\mathrm{gas}}\right)$. j) Imaginary refractive index $(410 \mathrm{~nm})$.

parameters.

According to these results, we can assess that the base altitude and peak concentration, and the scale height to a lesser extent are the most important parameters, and the retrieval more informative about their values.
To address the importance of these most informative free parameters in both the nadir-viewing reflectivity and the limb-darkening, we made a new Minnaert fit to models computed at nominal values as well as $1-\sigma$ above and below the nominal result. In Fig. 16 we show how the average value of the values of $I / F_{0}$ and of $k$ for the different 


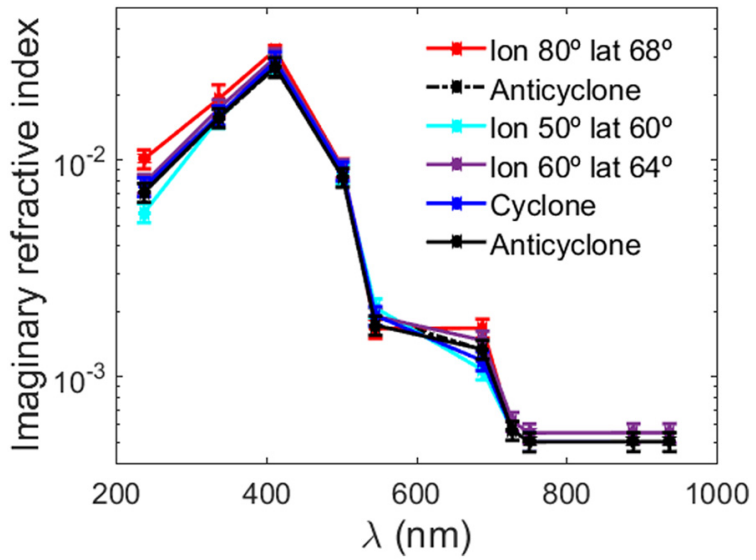

Fig. 15. The imaginary refractive indexes respect to the wavelength for six selected regions.

wavelengths varies as a given parameter is changed. From these results we observe that the most affected filters are F336W, FQ750N, FQ889N and FQ937N.

\section{Discussion}

We have found that in the region where ACA system is located, the optical thickness of the stratospheric and tropospheric hazes depends to a large extent on the latitude. This variation is not so pronounced in the lower cloud. As a general rule, we observe that the pressure of the base of the tropospheric haze decreases northwards from north $700 \pm 100$ mbar to $300 \pm 100$ mbar.

According to our modelling there are no significant differences between the anticyclones (A) and cyclones (C) either in the stratospheric haze (at pressures levels above tropopause $\sim 60-100 \mathrm{mbar}$ ) or in the lower cloud (at 1-1.4 bar).

If we compare the reflectivity of the three vortices with the surrounding regions in the range of selected latitudes, we find that in the FQ889N and FQ937N filters the cyclone has low brightness relative to the surroundings, but turns bright in F336W, consistent with low particle density in the tropospheric haze and deeper clouds. A similar behavior has been found in other cyclones (Sánchez-Lavega et al., 2006;
Table 4

Improvement factor of free parameters.

\begin{tabular}{lc}
\hline & Improvement factor \\
\hline Stratospheric haze & \\
$\tau_{\text {str }}(0.9 \mu \mathrm{m})$ & $2 \%$ \\
Tropospheric haze & \\
$\mathrm{P}_{\text {bot }}(\mathrm{mbar})$ & $92 \%$ \\
$\mathrm{~N}($ part $/ \mathrm{cm} 3)$ & $95 \%$ \\
Haerosol/Hgas & $15 \%$ \\
$\mathrm{r}_{\text {eff }}(\mu \mathrm{m})$ & $12 \%$ \\
$\sigma_{\text {eff }}(\mu \mathrm{m})$ & $3 \%$ \\
$\mathrm{~m}_{\mathrm{i}}(\mathrm{lambda})$ & $10 \%$ \\
$\mathrm{Cloud}$ & \\
$\tau_{\text {cloud }}(0.9 \mu \mathrm{m})$ & $5 \%$ \\
\hline
\end{tabular}

Baines et al., 2009). This is in agreement with our result since we found a minimum of optical thickness in the tropospheric haze $(\sim 10 \pm 2)$ and a lower concentration of particles $\left(\sim 55 \pm 10\right.$ particles $\left./ \mathrm{cm}^{3}\right)$. On the other hand, anticyclones are brighter than their surroundings in the FQ889N and FQ937N filters and darker in the F336N filter. This situation is associated to two regions with greater optical thickness $(\sim 25 \pm 2)$ and a higher concentration of particles $(\sim 80 \pm 10$ particles $/ \mathrm{cm}^{3}$ ). Similar observations have been made by Roman et al. (2013) in long-lived cyclones located at $51^{\circ} \mathrm{S}$ and by del Río-Gaztelurrutia et al. (2010), who investigated the vertical structure as well as the winds and dynamics.

The particle properties in both regions (effective radius and imaginary refractive index) are similar within our model sensitivity, indicating that for the ACA, no particular difference in microphysics processes and chromophore agents exit. The main difference in the cloud structure between the A and C occurs in the tropospheric haze and affects only to the particle number density and vertical thickness, higher in A than in C. No difference is found between A and C in the base location of this haze, in both cases at $\sim 500 \mathrm{mbar}$. The excess of tropospheric haze particles in A compared to $\mathrm{C}$ can be due to a higher ammonia ice condensation in the anticyclones, due to differences in temperature or in the vapor abundance at this level. For Jupiter's anticyclones, dynamical modelling proposes the existence of a cold core above the main cloud deck (Marcus et al., 2012) probably favoring haze formation. This could be also the situation for Saturn anticyclones. On the other hand, the fact that particle density is lower in C than in A

Table 3

Region 1 corresponds to longitude $80^{\circ}$ - latitude $60^{\circ} \mathrm{N}$ and region 2 corresponds to longitude $80^{\circ}$ - latitude $68^{\circ} \mathrm{N}$.

\begin{tabular}{|c|c|c|c|c|}
\hline & Anticyclone & Cyclone & Region 1 & Region 2 \\
\hline \multicolumn{5}{|l|}{ Stratospheric haze } \\
\hline$\tau_{\text {str }}(0.9 \mu \mathrm{m})$ & $0.015 \pm 0.01$ & $0.015 \pm 0.01$ & $0.01 \pm 0.01$ & $0.025 \pm 0.01$ \\
\hline \multicolumn{5}{|l|}{ Tropospheric haze } \\
\hline $\mathrm{z}(\mathrm{km})$ & $40 \pm 5$ & $50 \pm 5$ & $20 \pm 5$ & $60 \pm 5$ \\
\hline$P_{\text {bot }}$ (mbar) & $550 \pm 50$ & $500 \pm 50$ & $750 \pm 50$ & $300 \pm 50$ \\
\hline $\mathrm{N}\left(\right.$ part $\left./ \mathrm{cm}^{3}\right)$ & $78 \pm 10$ & $55 \pm 10$ & $100 \pm 10$ & $50 \pm 10$ \\
\hline $\mathrm{H}(\mathrm{km})$ & $18 \pm 2$ & $18 \pm 2$ & $23 \pm 2$ & $17 \pm 2$ \\
\hline$\tau_{\text {trop }}(0.9 \mu \mathrm{m})$ & $25 \pm 2$ & $10 \pm 2$ & $28 \pm 2$ & $10 \pm 2$ \\
\hline Thickness tropospheric haze $(\mathrm{km})$ & $70 \pm 2$ & $50 \pm 2$ & $78 \pm 2$ & $35 \pm 2$ \\
\hline $\mathrm{r}_{\mathrm{eff}}(\mu \mathrm{m})$ & $0.14 \pm 0.1$ & $0.15 \pm 0.1$ & $0.15 \pm 0.1$ & $0.15 \pm 0.1$ \\
\hline$\sigma_{\text {eff }}(\mu \mathrm{m})$ & $0.05 \pm 0.01$ & $0.05 \pm 0.01$ & $0.05 \pm 0.01$ & $0.05 \pm 0.01$ \\
\hline $\mathrm{m}_{\mathrm{i}}(225 \mu \mathrm{m})$ & $7 \pm 0.1 \mathrm{E}-03$ & $7 \pm 0.1 \mathrm{E}-03$ & $6 \pm 0.1 \mathrm{E}-03$ & $12 \pm 0.1 \mathrm{E}-03$ \\
\hline $\mathrm{m}_{\mathrm{i}}(336 \mu \mathrm{m})$ & $15 \pm 0.1 \mathrm{E}-03$ & $15 \pm 0.1 \mathrm{E}-03$ & $16 \pm 0.1 \mathrm{E}-03$ & $18 \pm 0.1 \mathrm{E}-03$ \\
\hline $\mathrm{m}_{\mathrm{i}}(410 \mu \mathrm{m})$ & $26 \pm 0.1 \mathrm{E}-03$ & $26 \pm 0.1 \mathrm{E}-03$ & $26 \pm 0.1 \mathrm{E}-03$ & $32 \pm 0.1 \mathrm{E}-03$ \\
\hline $\mathrm{m}_{\mathrm{i}}(502 \mu \mathrm{m})$ & $7 \pm 0.1 \mathrm{E}-03$ & $7 \pm 0.1 \mathrm{E}-03$ & $8 \pm 0.1 \mathrm{E}-03$ & $9 \pm 0.1 \mathrm{E}-03$ \\
\hline $\mathrm{m}_{\mathrm{i}}(547 \mu \mathrm{m})$ & $1.7 \pm 0.1 \mathrm{E}-03$ & $1.7 \pm 0.1 \mathrm{E}-03$ & $3 \pm 0.1 \mathrm{E}-03$ & $1.6 \pm 0.1 \mathrm{E}-03$ \\
\hline $\mathrm{m}_{\mathrm{i}}(689 \mu \mathrm{m})$ & $1.3 \pm 0.1 \mathrm{E}-03$ & $1.3 \pm 0.1 \mathrm{E}-03$ & $1.3 \pm 0.1 \mathrm{E}-03$ & $1.7 \pm 0.1 \mathrm{E}-03$ \\
\hline $\mathrm{m}_{\mathrm{i}}(727 \mu \mathrm{m})$ & $5 \pm 0.1 \mathrm{E}-04$ & $5 \pm 0.1 \mathrm{E}-04$ & $5 \pm 0.1 \mathrm{E}-04$ & $5 \pm 0.1 \mathrm{E}-04$ \\
\hline $\mathrm{m}_{\mathrm{i}}(750 \mu \mathrm{m})$ & $5 \pm 0.1 \mathrm{E}-04$ & $5 \pm 0.1 \mathrm{E}-04$ & $5 \pm 0.1 \mathrm{E}-04$ & $5 \pm 0.1 \mathrm{E}-04$ \\
\hline $\mathrm{m}_{\mathrm{i}}(889 \mu \mathrm{m})$ & $5 \pm 0.1 \mathrm{E}-04$ & $5 \pm 0.1 \mathrm{E}-04$ & $5 \pm 0.1 \mathrm{E}-04$ & $5 \pm 0.1 \mathrm{E}-04$ \\
\hline $\mathrm{m}_{\mathrm{i}}(937 \mu \mathrm{m})$ & $5 \pm 0.1 \mathrm{E}-04$ & $5 \pm 0.1 \mathrm{E}-04$ & $5 \pm 0.1 \mathrm{E}-04$ & $5 \pm 0.1 \mathrm{E}-04$ \\
\hline \multicolumn{5}{|l|}{ Cloud } \\
\hline$\tau_{\text {cloud }}(0.9 \mu \mathrm{m})$ & $9 \pm 2$ & $9.5 \pm 2$ & $9.5 \pm 2$ & $8.8 \pm 2$ \\
\hline
\end{tabular}


a)

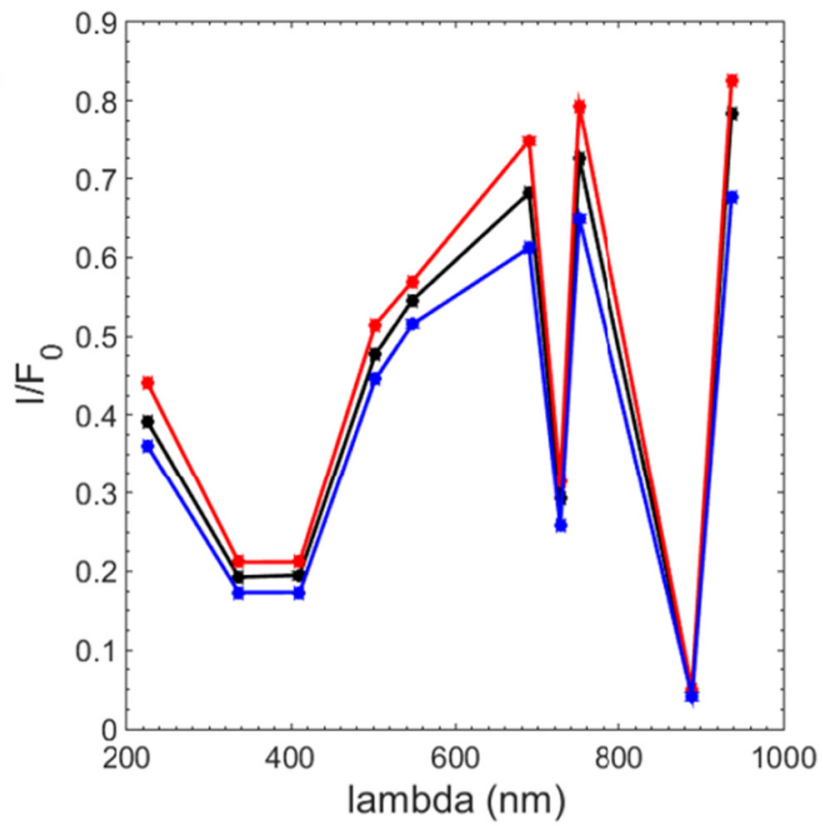

b)

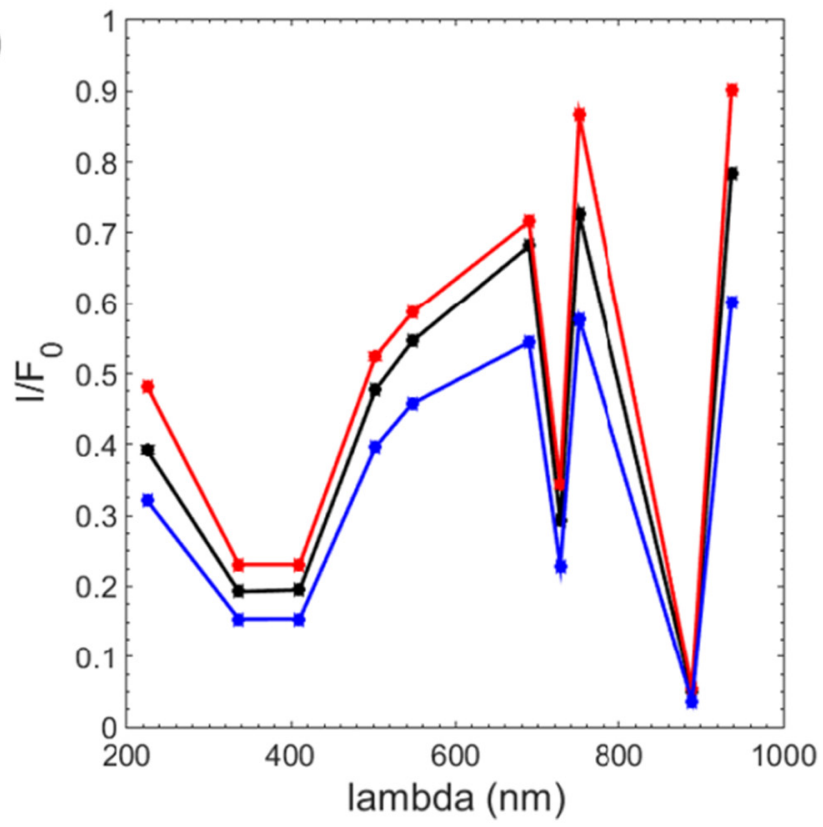

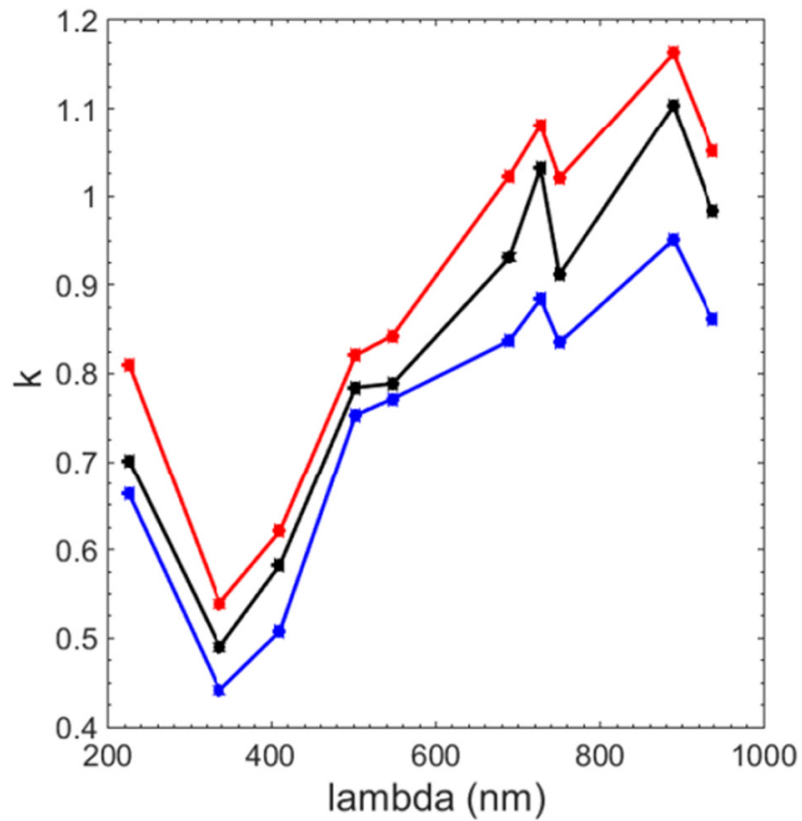

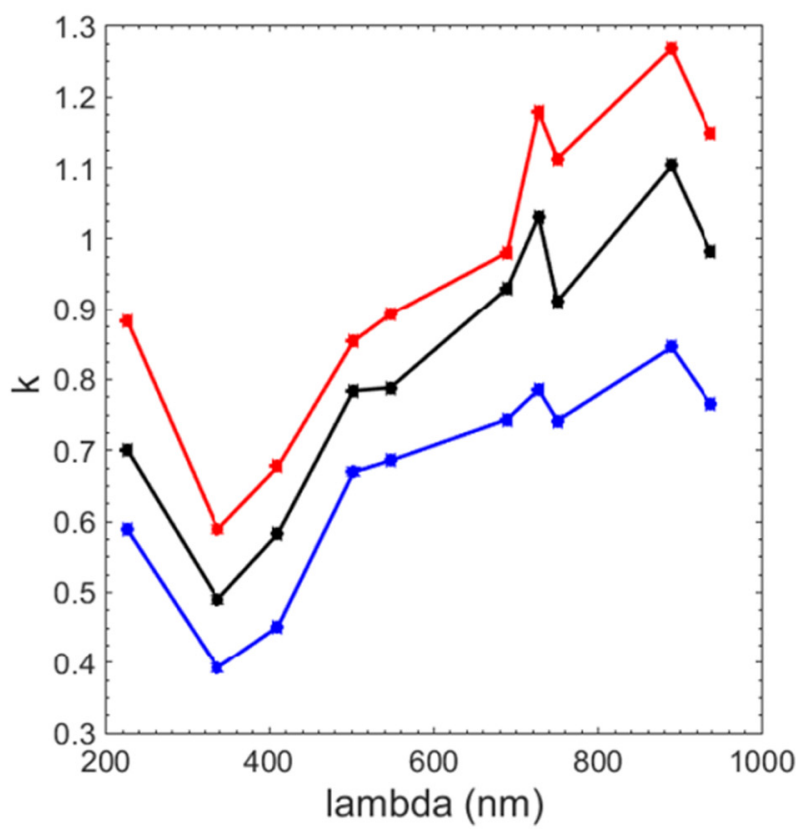

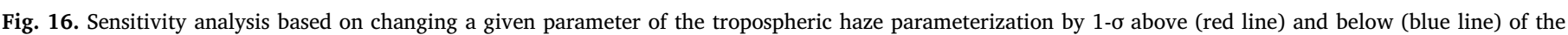

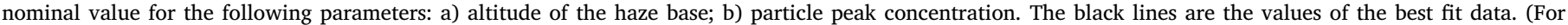
interpretation of the references to color in this figure legend, the reader is referred to the web version of this article.)

could also be due to vertical motions, with subsidence in $\mathrm{C}$ at the tropospheric haze, also suggested in a previous work (del RíoGaztelurrutia et al., 2010). Note that this is at odds with the behavior that dominates Earth's vortices: subsidence and ample cloud free areas in anticyclones, and cloudy extratropical and tropical cyclones where cloud formation by baroclinic frontal systems and massive moist convection occurs.

\section{Conclusions}

We report a photometric analysis and radiative transfer modelling of a triple vortex (ACA system) in Saturn's atmosphere using images taken with the HST/WFC3 at a spectral range from $225 \mathrm{~nm}$ to $937 \mathrm{~nm}$, including an intermediate and a deep methane absorption band. We retrieve the vertical distribution and properties of the upper cloud and hazes at a region which includes this ACA system, covering a range of latitudes from $55^{\circ} \mathrm{N}$ to $69^{\circ} \mathrm{N}$. Below we list the most important conclusions.

- Most atmospheric parameters seem to be zonally homogenous except in the region of the ACA system, where a few of them have significant variations.

- These variations correspond to characteristic parameters of the tropospheric haze, in particular, the particle number density and the base height.

- The optical thickness of the stratospheric haze is quite homogeneous in longitude while increasing with latitude. The optical thickness of the cloud is nearly constant

- The optical thickness of the tropospheric haze increases from $\tau_{\text {trop }}$ $\sim 28 \pm 2$ at $55^{\circ} \mathrm{N}$ up to $\tau$ trop $\sim 35 \pm 2$ at $61^{\circ} \mathrm{N}$, and then decreases 
down to $\tau_{\text {trop }} \sim 10 \pm 2$ at $69^{\circ} \mathrm{N}$. The greatest variability is found in the range of latitudes of the ACA system. At the anticyclones $\tau_{\text {trop }}$ $\sim 20 \pm 2$, while in the cyclone $\tau_{\text {trop }} \sim 15 \pm 2$.

- Both the anticyclones and the cyclone display a base pressure of the tropospheric haze $(\sim 550 \pm 50 \mathrm{mbar}$ with a greater thickness $\sim 70 \pm 2 \mathrm{~km}=3 \mathrm{H}$ and $\sim 500 \pm 50 \mathrm{mbar}$ with a thickness of $50 \pm 2 \mathrm{~km}=2 \mathrm{H}$ ), lower than the base pressures of the regions at lower latitudes $(750 \pm 50 \mathrm{mbar}$ with a thickness $78 \pm 2 \mathrm{~km}=3.25 \mathrm{H}$ to $55^{\circ} \mathrm{N}$ ) and higher than the base pressures at higher latitudes $(300 \pm 50 \mathrm{mbar}$ with a thickness $35 \pm 2 \mathrm{~km}=1.5 \mathrm{H}) . \mathrm{H}$ is the atmospheric scale height.

- The maximum particle number density is higher in the anticyclones $\left(\sim 78 \pm 10\right.$ particles $\left./ \mathrm{cm}^{3}\right)$ than in the cyclonic region $(\sim 50 \pm 10$ particles $/ \mathrm{cm}^{3}$ ).

- The low values of optical thickness, the concentrations of particles found, as well as the base height of the tropospheric haze in the cyclone suggest that it is a subsidence region.

- The vortices show no significative variations in the scale height, particle size or refractive indices of the haze.

- The properties of the anticyclones and cyclone are compatible with the general picture of upwelling in the former and downwelling in the latter.

\section{Acknowledgments}

This work was supported by the Spanish project AYA2015-65041-P (MINECO/FEDER, UE) and Grupos Gobierno Vasco IT-765-13.

\section{References}

Baines, K.H., Delitsky, M.L., Momary, T.W., Brown, R.H., Buratti, B.J., Clark, R.N., Nicholson, P.D., 2009. Storm clouds on Saturn: lightning-induced chemistry and associated materials consistent with Cassini/VIMS spectra. Planet. Space Sci. 57, 1650-1658. https://doi.org/10.1016/j.pss.2009.06.025.

De Pater, I., Lissauer, J., 2001. Planetary Science. Cambridge University Press, Cambridge, U.K.

Del Genio, A.D., Achterberg, R.K., Baines, K.H., Flasar, F.M., Read, P.L., Sánchez-Lavega, A., Showman, A.P., 2009. Saturn atmospheric structure and dynamics. In: Dougherty, M., Esposito, L., Krimigis, T. (Eds.), Saturn From Cassini-Huygens. Springer-Verlag, pp. 113-159 (Chapter 6).

del Río-Gaztelurrutia, T., Legarreta, J., Hueso, R., Pérez-Hoyos, S., Sánchez-Lavega, A. 2010. A long-lived cyclone in Saturn's atmosphere: observations and models. Icarus 209, 665-681. https://doi.org/10.1016/j.icarus.2010.04.002.

del Río-Gaztelurrutia, T., Sánchez-Lavega, A., Antuñano, A., Legarreta, J., GarcíaMelendo, E., Sayanagi, K.M., Hueso, R., Wong, M., Pérez-Hoyos, S., Rojas, J.F., Simon, A.A., de Pater, I., Blalock, J., Barry, T., 2018. A planetary-scale disturbance in a long living three vortex coupled system in Saturn's atmosphere. Icarus 302, 499-513.

Dressel, L., 2019. Wide Field Camera 3 Instrument Handbook, Version 11.0. STScI, Baltimore.

Fletcher, L.N., Irwin, P.G.J., Teanby, N.A., Orton, G.S., Parrish, P.D., de Kok, R., Howett, C., Calcutt, S.B., Bowles, N., Taylor, F.W., 2007. Characterising Saturn's vertical temperature structure from Cassini/CIRS. Icarus 189, 457-478.

García-Melendo, E., Pérez-Hoyos, S., Sánchez-Lavega, Hueso, R., 2011. Saturn's zonal wind profile in 2004-2009 from Cassini ISS images and its long-term variability. Icarus 215, 62-74. https://doi.org/10.1016/j.icarus.2011.07.005.

García-Melendo, E., Sánchez-Lavega, A., Hueso, R., 2007. Numerical models of Saturn's long-lived anticyclones. Icarus 191, 665-677. https://doi.org/10.1016/j.icarus.2007. 05.020 .

Hansen, J.E., Travis, L.D., 1974. Light scattering in planetary atmospheres. Space Sci. Rev. 16, 527-610.

Ingersoll, A.P., Beebe, R.F., Conrath, B.J., Hunt, G.E., 1984. Structure and dynamics of Saturn's atmosphere. In: Gehrels, T., Matthews, M.S. (Eds.), Saturn. Univ. of Arizona
Press, Tucson, pp. 195-238.

Ingersoll, A.P., Ewald, S.P., Sayanagi, K.M., Blalock, J.J., 2018. Saturn's atmosphere at 1-10 kilometer resolution. Geophys. Res. Lett. 45. https://doi.org/10.1029/ 2018GL079255.

Irwin, P.G.J., Teanby, N.A., de Kok, R., et al., 2008. The NEMESIS planetary atmosphere radiative transfer and retrieval tool. J. Quant. Spectrosc. Radiat. Transf. 109, 1136-1150.

Irwin, P.G.J., Fletcher, L.N., Read, P.L., et al., 2015. Spectral analysis of Uranus' 2014 bright storm with VLT/SINFONI. Icarus 264, 72-89.

Karkoschka, E., Tomasko, M.G., 2005. Saturn's vertical and latitudinal cloud structure 1991-2004 from HST imaging in 30 filters. Icarus 179, 195-221.

Karkoschka, E., Tomasko, M.G., 2010. Methane absorption coefficients for the Jovian planets from laboratory, Huygens, and HST data. Icarus 205, 674-694.

Lucarini, V., Saarinen, J.J., Peiponen, K.E., Vartiainen, E.M., 2005. Kramers-Kronig Relations in Optical Materials Research. Springer-Verlag Berlin Heidelberg, Germany.

Marcus, P.S., Asay-Davis, X., Wong, M.H., de Pater, I., 2012. Jupiter's new red oval: dy namics, color, and relationship to jovian climate change. J. Heat Transf. 135 (1), 011007 (9 pages).

Ortiz, J.L., Moreno, M., Molina, A., 1996. Saturn 1991-1993: clouds and hazes. Icarus $119,53-66$.

Pérez-Hoyos, S., Sánchez-Lavega, A., French, R.G., Rojas, J.F., 2005. Saturn's cloud structure and temporal evolution from ten years of Hubble space telescope images (1994-2003). Icarus 176, 155-174.

Pérez-Hoyos, S., Sanz-Requena, J.F., Sánchez-Lavega, A., Irwin, P.G.J., Smith, A., 2016. Saturn's tropospheric particles phase function and spatial distribution from Cassini ISS 2010-11 observations. Icarus 277, 1-18.

Roman, M.T., Banfield, D., Gierasch, P.J., 2013. Saturn's cloud structure from Cassini ISS. Icarus 225, 93-110.

Sánchez-Lavega, A., Rojas, J.F., Sada, P.V., 2000. Saturn's zonal winds at cloud level. Icarus 147, 405-420. https://doi.org/10.1006/icar.2000.6449.

Sánchez-Lavega, A., Hueso, R., Pérez-Hoyos, S., Rojas, J.F., French, R.G., 2004. Saturn's cloud morphology and zonal winds before the Cassini encounter. Icarus 170, 519-523. https://doi.org/10.1016/j.icarus.20 04.05.0 02.

Sánchez-Lavega, A., Hueso, R., Pérez-Hoyos, S., Rojas, J.F., 2006. A strong vortex in Saturn's south pole. Icarus 184, 524-531.

Sánchez-Lavega, A., Fisher, G., Fletcher, L.N., Garcia-Melendo, E., Hesman, B., PerezHoyos, S., Sayanagi, K., Sromovsky, L., 2019a. In: Baines, K.H., Flasar, F.M., Krupp, N., Stallard, T.S. (Eds.), The Great Storm of 2010-2011. Cambridge University Press, 978-1-107-10677-2, pp. 377-416 Chapter 13 of the book Saturn in the 21st Century.

Sánchez-Lavega, A., García-Melendo, E., Legarreta, J., Hueso, R., del Río-Gaztelurrutia, T., Sanz-Requena, J.F., Pérez-Hoyos, S., Simon, A.A., Wong, M.H., Soria, M., GómezForrellad, J.M., Barry, T., Delcroix, M., Sayanagi, K.M., Blalock, J.J., Gunnarson, J.L., Dyudina, U., Ewald, S., 2019b. A complex storm system and a planetary-scale disturbance in Saturn's north polar atmosphere in 2018. Nat. Astron (submitted).

Sanz-Requena, J.F., Pérez-Hoyos, S., Sánchez-Lavega, A., et al., 2012. Cloud structure of Saturn's 2010 storm from ground-based visual imaging. Icarus 219, 142-149.

Sanz-Requena, J.F., Pérez-Hoyos, S., Sánchez-Lavega, A., Antuñano, A., Iwing, P., 2018. Haze and cloud structure of Saturn's North Pole and Hexagon Wave from Cassini/ISS imaging. Icarus 305, 284-300.

Sayanagi, K., West, R., Baines, K., Fletcher, L., Read, P., Dyudina, U., 2019. Saturn's poles: vortices, hexagons, and auroral aerosols. Chapter 12 of the book In: Baines, K.H., Flasar, F.M., Krupp, N., Stallard, T.S. (Eds.), Saturn in the 21st Century. Cambridge University Press, 978-1-107-10677-2, pp. 337-376.

Smith, B.A., 1981. Encounter with Saturn: Voyager 1 imaging results. Science 212, 163-191. https://doi.org/10.1126/science.212.4491.163.

Smith, B.A., 1982. A new look at the Saturn system: the Voyager 2 images. Science 215, 505-537. https://doi.org/10.1126/science.215.4532.504.

Sromovsky, L.A., Baines, K.H., Fry, P.M., 2013. Saturn's Great Storm of 2010-2011: evidence for ammonia and water ices from analysis of VIMS spectra. Icarus 226, 402-418.

Trammell, H.J., Li, L., Jiang, X., Pan, Y., Smith, M.A., Bering, E.A., Hörst, S.M., Vasavada, A.R., Ingersoll, A.P., Janssen, M.A., West, R.A., Porco, C.C., Li, C., Si- mon, A.A., Baines, K.H., 2016. Vortices in Saturn's Northern Hemisphere (2008-2015) observed by Cassini ISS. J. Geophys. Res. Planets 121, 1814-1826. https://doi.org/10.1002/ 2016JE005122.

Vasavada, A.R., Hörst, S.M., Kennedy, M.R., Ingersoll, A.P., Porco, C.C., Del Genio, A.D., West, R.A., 2006. Cassinin imaging of Saturn: southern hemisphere winds and vortices. J. Geophys. Res. 111, E05004.

West, R.A., Baines, K.H., Karkoschka, E., Sánchez-Lavega, A., 2009. Clouds and aerosols in Saturn's atmosphere. In: Dougherty, M.K., Esposito, L.W., Krimigis, S.M. (Eds.), Saturn From Cassini-Huygens. Springer, Netherlands, pp. 161-179. 